\title{
柔性电子喷印制造: 材料、工艺和设备
}

\author{
尹周平*，黄永安*，布宁斌，王小梅，熊有伦 \\ 华中科技大学机械学院, 数字制造装备与技术国家重点实验室, 武汉 430074 \\ * 联系人, E-mail: yinzhp@mail.hust.edu.cn; yahuang@ @ust.edu.cn
}

2009-10-20 收稿, 2010-02-05 接受

国家自然科学基金(50705035, 50625516)和国家重点基础研究发展计划(2009CB724204)资助项目

\begin{abstract}
摘要喷印技术直接将功能材料沉积到基板上形成图案, 有望成为高性能柔性电子的主流制 造工艺之一。目前，喷印技术面临材料、工艺和设备等诸多挑战。本文讨论了无机、有机以及 纳米复合喷印材料的电学性能, 分析了打印性能与黏度、表面张力、蒸发率等关系. 压电、热 泡等传统喷印可实现微米级分辨率图案化, 而电喷涂、电纺丝、电喷印等电流体动力喷印可实 现纳米级分辨率图案化, 如何通过多场调控提高其喷印过程操控性至为关键. 讨论了电流体动 力喷印设备实现关键技术, 包括液滴操控、喷嘴设计、卷到卷输送等。最后展望了柔性电子喷 印制造需研究并解决的关键科学技术问题.

关键词

柔性电子

纳米制造

有机薄膜晶体管 微/纳图案化

喷印

电流体动力学 卷到卷
\end{abstract}

\section{1 柔性电子}

柔性电子, 又称之为打印电子或有机电子, 是将 有机/无机电子器件沉积在柔性基板上形成电路的技 术. 由于柔性电子性能与传统微电子相当, 且具有便 携性、透明、轻质、伸展/弯曲，以及易于快速大面积 打印等特点, 产生了许多新的应用 ${ }^{[1 ~ 4]}$, 如柔性显示

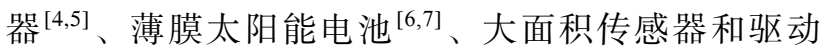
器 ${ }^{[8,9]}$ 等(如图 1 所示). 柔性电子是一个新兴开放的研 究领域, 随着学科交叉的加深, 新的应用不断出现, 在新型电子材料、微纳制造工艺和设备等方面不断出 现新的研究问题.

柔性电子区别于传统微电子的最大特点是其柔 性. 聚合物有机材料与塑料基板自然兼容，一般被认 为非常适合于柔性电子的应用, 但是用其制作器件 的电学性能不够理想, 这就促使利用无机材料制备 柔性电子 ${ }^{[10]}$. 无机材料的断裂应变比较小, 如何设 计制造具有良好弯曲和伸缩性能的器件成为难点. 最常用的方法是将用于制作半导体、导体或者绝缘体 的无机材料制备成薄膜, 以减小弯曲或伸缩状态下
产生的应变. 目前, 对无机材料薄膜的弯曲、伸缩、 延展和断裂等行为的力学特性进行了大量研究. 文 献[11]对塑料基板上无机电子材料的弯曲性开展了 理论和实验研究, 给出了界面剪切和剥离应力. 文 献[12 16]提出利用屈曲行为设计波纹结构的方法, 可实现柔性电子完全可逆拉伸/压缩, 而电路材料本 身没有产生明显的应变. 屈曲波纹可在微纳米尺度 进行控制, 得到 $100 \mathrm{~nm} 100 \mu \mathrm{m}$ 的几何尺寸结构, 其 伸缩性可通过控制屈曲波纹的方向、幅值和波长进行 调整. 单一的硅/金属薄膜一般在应变超过 1\% 2\%时 发生断裂，然而当其黏合到塑料基板上时可实现 10 倍于断裂应变的塑性变形并保持导电性 ${ }^{[14,19,20]}$. 文 献[21]对柔性电子系统及其机械特性进行了评述. 文 献[22,23]研究了基板的温变特性对柔性电子结构可 靠性的影响, 给出了屈曲结构的临界工作温度.

柔性电子制造过程通常包括: 材料制备 $\rightarrow$ 沉积 $\rightarrow$ 图案化 $\rightarrow$ 封装, 可通过卷到卷(R2R)基板输送进行 集成, 如图 2 所示 ${ }^{[24]}$. 柔性电子制造主要关注生产成 本、生产效率、可实现的特征尺寸，以及有机材料的 相容性等因素. 近年来，由于活性材料及其图案化技

英文版见: Yin Z P, Huang Y A, Bu N B, et al. Inkjet printing for flexible electronics: Materials, processes and equipments. Chinese Sci Bull, 2010, 55, doi: 10.1007/s11434-010-3251-Z 


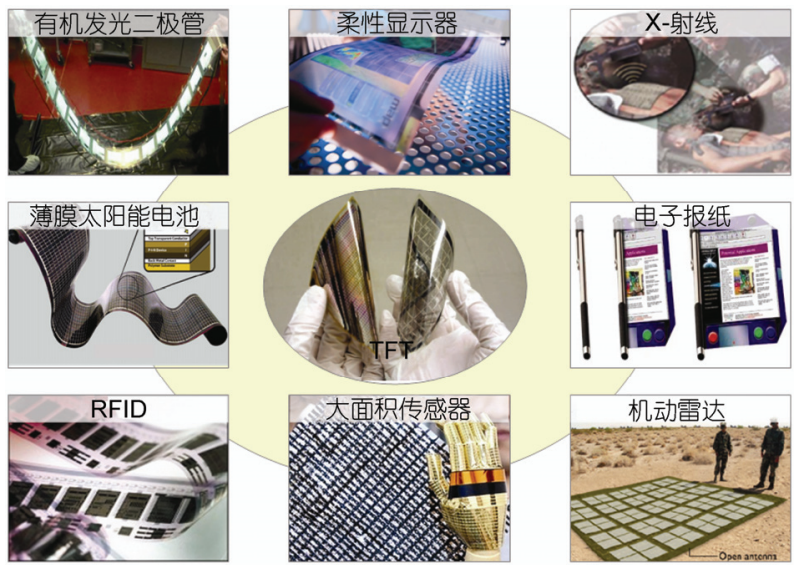

图 1 基于柔性 TFT 阵列技术的一系列应用

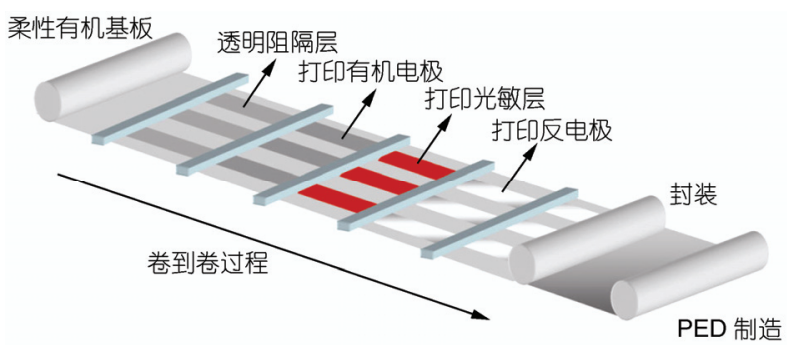

图 2 基于 R2R 技术的柔性电子制造工艺过程示意图 ${ }^{[24]}$

术的突破, 柔性电子制造技术得到了长足的发展.

柔性电子制造的核心是薄膜晶体管(TFT)制造, 其关键制造技术是制作源漏极间沟道长度的高分辨 率图案化技术, 直接影响输出电流、开关速度等器件 性能. 在有机半导体图案化过程中, 特别需要消除寄 生漏电和减少串音, 以确保高的开关比. 大多数应用 要求有机薄膜晶体管(OTFT)沟道长度小于 $10 \mu \mathrm{m}$. 现有的图案化技术包括光刻、荫罩、打印(微接触印 制和喷印)等, 具体比较见表 1 . 光刻等能量束技术在 微电子器件图案化中得到广泛应用, 分辨率高, 但因 其工艺过程复杂、设备昂贵、溶剂和显影剂无法用于 塑料基板, 加之耗时费料、仅适用于小面积图案化 ${ }^{[25]}$, 在刻蚀底层时环境要求苛刻, 去除光刻胶时会破坏 有机电子材料的活性和聚合物基板 ${ }^{[24]}$ 等, 在柔性电 子制造应用中受限 ${ }^{[26]}$. 荫罩技术为“干”工艺，可避免 溶剂破坏有机半导体，但分辨率有限.

打印技术在同一个步骤中同时实现功能材料沉 积和图案化, 主要方法有: (1) 将完整的电路转移并 粘贴到柔性基板上，如传印(图章)；(2) 直接在柔性 基板上制备电路, 如喷印和微接触印制(软刻蚀) ${ }^{[26]}$.
表 1 主要图案化技术比较

\begin{tabular}{ccccc}
\hline & 光刻 & \multicolumn{3}{c}{ 荫罩 } \\
\hline 微接触印制 & 喷印 \\
\hline 成本 & 极高 & 低 & 中等 & 低 \\
可制造面积 & 小 & 大 & 中等 & 大 \\
效率 & 低 & 高 & 高 & 高 \\
温度 & 高温高压 & 低温 & 中等 & 高温、低温均可 \\
掩膜 & 需要 & 需要 & 不需要 & 不需要 \\
分辨率 & 极高 & 低 & 高 & 高 \\
有机材料兼容性 & 差 & 良好 & 差 & 优异 \\
灵活性 & 差 & 差 & 差 & 好, 数字化刻蚀 \\
R2R 兼容性 & 差 & 中等 & 良好 & 良好 \\
材料消耗 & 严重 & 中等 & 低 & 低 \\
环境要求 & 净化空间, 隔振 & 低 & 中等 & 低 \\
工艺步骤 & 多步 & 多步 & 多步 & 单步 \\
实现模式 & 非接触式 & 接触式 & 接触式 & 非接触式 \\
\hline
\end{tabular}

在传印方法中，首先通过标准光刻方法在硅晶片或 玻璃板上制备整个结构, 然后转移到柔性基板上制 造出高性能器件. 由于应用光刻和高温沉积技术, 传 印技术只能制造小面积器件, 且加工成本高 ${ }^{[1]}$. 微接 触印制可制造出多级图案用于掩模, 可与 $R 2 R$ 批量 化制造技术集成. 通常一个母版可制造 100 个以上的 图章, 每个图章又可实现 3000 个以上的印记, 图章 的成本相对较低 ${ }^{[1]}$, 可以每秒数厘米的速度制作 60 $\mathrm{nm}$ 高分辨率图案, 但实现多层图案比较困难 ${ }^{[27]}$. 微 接触印制可用于非晶硅、多晶硅及 TMOS 等多种材 料, 但难以直接用于有机材料刻蚀. 兰红波等人 ${ }^{[28]}$ 对纳米压印刻蚀模具技术的研究进展及其发展趋势 进行了详细的论述和分析.

柔性电子理想的图案化工艺应满足：低成本、大 面积、批量化工艺、低温、“加”式、非接触式、可实 时调整、三维结构化、易于多层套准、可打印有机物/ 
无机材料等. 从表 1 可知, 喷印是一种无接触、无压 力、无印版的印刷复制技术, 它具有无版数码印刷的 特征, 在室温下将溶液直写实现数字化柔性印刷, 简 化了制造过程 ${ }^{[29,30]}$. 利用溶液化的半导体和金属材 料取代传统的真空沉积材料, 可有效减低成本 ${ }^{[2]}$. 喷 印还具有以下优势: (1) 图案质量不受光刻焦距限制, 可在非平面表面甚至深沟结构上进行图案化 ${ }^{[30]}$; (2) 与有机/无机材料的良好兼容性; (3) 直接利用 $\mathrm{CAD} /$ $\mathrm{CAM}$ 数据加工器件, 可实现大面积动态对准和实时 调整；(4) 作为非接触式图案化技术，可有效减少瑕 疪，并可利用虚拟掩模补偿层间变形、错位等缺陷; (5) 无需物理掩模的按需打印(DOD)技术; (6) 可实 现复杂三维微结构的快速设计与加工, 并可通过基 于软件打印控制系统进行图形的快速更改. 本文主 要讨论柔性电子喷印制造技术在材料、工艺和设备等 方面的研究进展与发展趋势.

\section{2 喷印材料}

\section{1 材料可打印性}

如图 3 所示, 典型 OLED 器件结构包括柔性基底 层、透明阻隔层、电极层、半导体层和发光层等 ${ }^{[24]}$. 利 用喷印实现器件的图案化要求功能墨液除了具备电 子迁移率、电压开关比、阈值电压稳定性等 ${ }^{\left[{ }^{[1,32]}\right.}$ 电性 能外, 还须具备可打印性：(1) 墨液无需掩膜能够稳 定、精确打印成图案; (2) 墨液须满足黏度、表面张

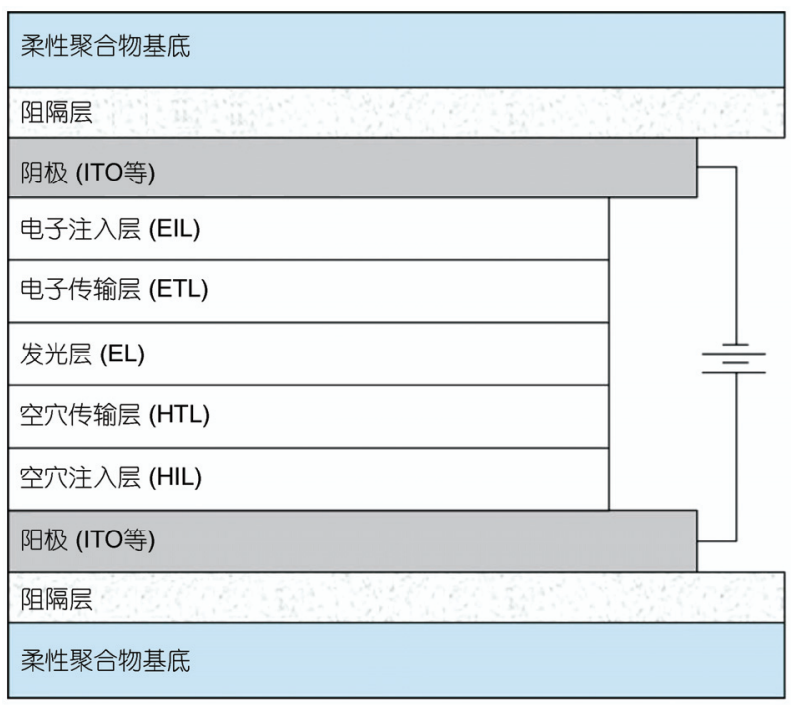

图 3 柔性 OLED 器件架构示意图 ${ }^{[24]}$
力等物理化学特性, 以获得最佳的打印图案、性能和 可靠性. 分子和纳米尺度结构将影响打印图案的属 性, 如形貌特征(表面粗䊅度、晶粒尺寸)、黏附性、 结构整合性、可溶性、以及化学和环境稳定性等. 这 些因素反过来又会影响柔性电子器件的性能, 特别 是电性能 ${ }^{[33]}$.

非牛顿流体的可打印性与其物理、流变性能相关, 影响因素除黏弹性、剪切与压缩状态的屈服应力 ${ }^{[26]}$ 外, 还包括速度 $(v)$ 、特征长度 $(\alpha)$ 、黍度 $(\eta)$ 、密度 $(\rho)$ 和表面张力 $(\gamma)$ 等 ${ }^{[34]}$. 常用雷诺数 (Reynolds, $N_{R e}=$ $v \alpha \rho / \eta$, 惯性与黏性力的比值)、韦伯数 (Weber, $N_{W e}=v^{2} \alpha \rho / \gamma$, 惯性量与毛细管力的平衡量 $)^{[35]}$ 、韦森 博格数 (Weissenberg, $W_{i}=\lambda v / \alpha, \lambda$ 是流体的特征驰 豫时间 $)^{[36]}$ 等无量纲量描述材料的黏弹性效应. 利用 雷诺数和韦伯数可得到如下公式:

$$
Z=\frac{(\alpha \rho \gamma)^{\frac{1}{2}}}{\eta}=\frac{N_{R e}}{\left(N_{W e}\right)^{\frac{1}{2}}} .
$$

墨液扩散行为取决于流体动力学特性 $\left(N_{R e}\right.$ 和 $N_{W e}$ ), 而可打印性取决于 Ohnesorge 数(与材料黏度、 表面张力和流体密度有关)的倒数 $Z$. 研究发现 ${ }^{[34]}$ : 当 $2<Z<4$ 时, 液滴拖尾的相对速度足够大, 可以追 上液滴头部, 并与其结合, 不发生尾部拉丝的零星断 裂现象; 当 $6<Z<13$ 时, 由于液滴表面张力使表面 面积最小化, 下落中出现主液滴拉丝零星断裂现象, 但尾部仍能保持足够的速度在最后拉丝反弹阶段实 现与主液滴再结合; 当 $Z>14$ 时, 在外驱动力作用下, 墨液极易发生喷射, 不存在明显黏性. 主液滴下落速 度较快时, 脱离尾部的卫星液滴无法追上主液滴实 现再结合. 较高鞭动动能和表面张力会使液滴发生 二次破裂，产生主液滴和永久性卫星液滴，所以当 $Z>14$ 时, 流体无法实现打印.

墨液表面张力通常应高于 $30 \mathrm{mN} / \mathrm{m}$, 外压力尽 可能低, 以防止墨液自发从喷嘴中滴落 ${ }^{[26]}$. 墨液黏 度应足够低(如表 2 所示), 使针管可在 $100 \mu \mathrm{s}$ 左右时 间内能重新填料 ${ }^{[27]}$. 墨液溶剂蒸发率应足够低, 避 免喷嘴堵塞. 要生成微米尺度直径的液滴, 需要足够 高的动能(如惠普 $51626 \mathrm{~A}$ 为 $20 \mu \mathrm{J}$ ) 和速度 (通常 1 10 $\mathrm{m} / \mathrm{s}$ ) 以克服液滴在喷嘴处保持半月形的界面能. 由于 压电或热汽泡薄膜所产生的能量有限，传统喷印技 术进行高黏度材料的打印非常困难，但电流体动力 
表 2 用于喷印溶液的典型参量

\begin{tabular}{cccccc}
\hline & 压电式 & 热气包式 & \multicolumn{1}{c}{ 电纺 } & 电喷 & 电喷 \\
& DOD & DOD & 丝 & 涂 & 印 \\
\hline 黏性 $/ \mathrm{Pa} \cdot \mathrm{s}$ & $<40$ & 1.28 & $7 \sim 175$ & 2.76 & $\sim 90$ \\
表面张力 $/ \mathrm{mN} \cdot \mathrm{m}^{-1}$ & $20 \sim 70$ & 取决于温度 & $15 \sim 64$ & $\sim 45$ & $\sim 50$ \\
\hline
\end{tabular}

打印(EHD)可实现高黏度流体的打印 ${ }^{[37]}$. 表 2 中列出 了喷印技术重要溶液参数 (黏性和表面张力)的大致 范围.

\section{2 无机喷印材料}

可打印无机材料包括金属和非金属, 如用作透 明导体的碳纳米管、用作半导体和绝缘体的过渡金属 氧化物、用作电极的金属纳米粒子等, 均可用于喷印.

( i ) 碳纳米管. 单壁碳纳米管/多壁碳纳米管 (SWCNTs/MWCNTs) 具有诸多优异物理性能 ${ }^{[38]}$, 如 卓越的导热性能、良好的机械强度、半导体/金属切 换性和优异的场发射效应. Fan 等人 ${ }^{[39]}$ 及 Tong 等人 ${ }^{[40]}$ 分别利用 MWCNTs 墨液和 MWCNTs 水溶液喷印制 备薄膜. 结果表明: 喷印第一层时, 两种墨液均不能 形成良好的导电网; 随着打印层的增加, MWCNTs 连续导电网逐渐形成, 电阻呈指数降低; 当 MWCNTs 墨液喷印到三层/MWCNTs 水溶液墨液喷
印到四层(不到 $100 \mathrm{~nm}$ 厚)时, 导电网足够紧凑和连 续, 涵盖纸上几乎所有的漏洞和空隙(如图 4 和 5 所 示), 此时, 即使进一步增加打印次数, 电阻难以继 续降低. Song 等人 ${ }^{[41]}$ 用纯 SWCNTs 的二甲基甲酰胺 溶液喷印出 SWCNTs 薄膜, 具有导电性和透明性. Mabrook 等人 ${ }^{[38]}$ 喷印出 SWCNTs 薄膜，薄膜遵循 Poole-Frenkel 导电模型, 电流与电压行为呈现非线性.

通过电纺丝已成功将碳纳米管嵌人聚合物纳米 纤维乙烯 $(\mathrm{PEO})^{[42]}$. 用两亲体, 无论是小分子 $(\mathrm{SDS}$, 十二烷基硫酸钠)还是聚合物 (Gum Arabic, 阿拉伯 胶), 可实现碳纳米管在水中的初步分散. Zhang 等 人 $^{[43]}$ 在 MWCNTs 表面接枝 Triton X-100 使其功能化, 改善了 MWCNTs 在聚丙烯腈(PAN)基溶液中的分散性. 利用二甲基甲酰胺溶液, 通过电纺丝制备含聚丙烯 腈和碳纳米管的复合材料纳米纤维, 直径约为 $100 \mathrm{~nm}$, 具有良好组织结构, 在平行电极之间实现单向分布. 图 6 是已嵌人纳米纤维内的表面功能化 MWCNTs 的 透射电子显微镜图.

(ii) 金属纳米粒子. 金 $(\mathrm{Au}) 、$ 银 $(\mathrm{Ag})$ 和铜 $(\mathrm{Cu})$ 等金属纳米粒子都可用于制备导电墨液, 主要类型 包括：（1）金属纳米颗粒悬浮液，金属粒子尺寸小, 烧结温度低 ${ }^{[44 ~ 46]} ;(2)$ 金属-有机前驱物溶液, 可在低
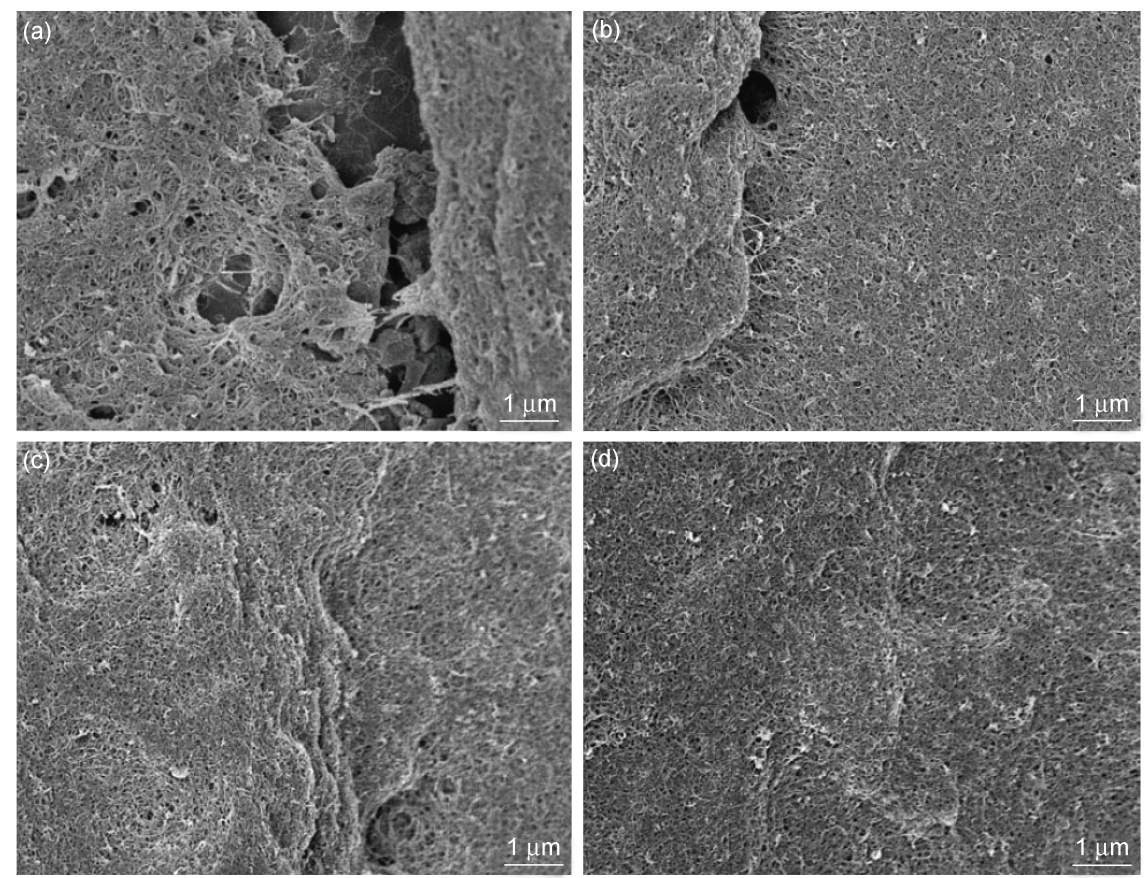

图 4 碳条纹的扫描电子显微镜图

(a) 打印 1 层; (b) 打印 2 层; (c) 打印 3 层和(d) 打印 4 层 ${ }^{[39]}$ 

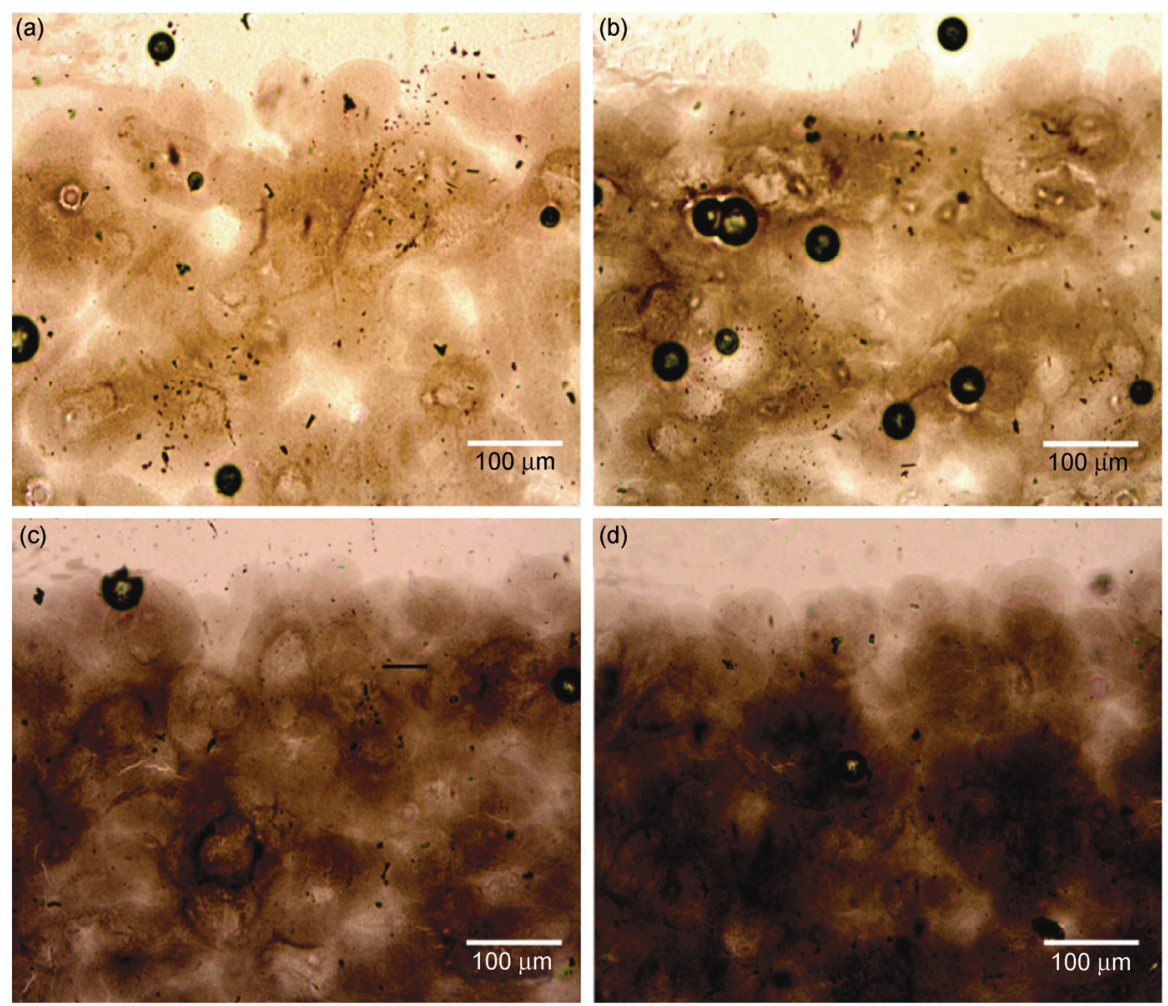

图 5 光学显微镜照片打印

(a)打印 1 层; (b)打印 2 层; (c)打印 3 层和(d)打印 4 层 ${ }^{[40]}$
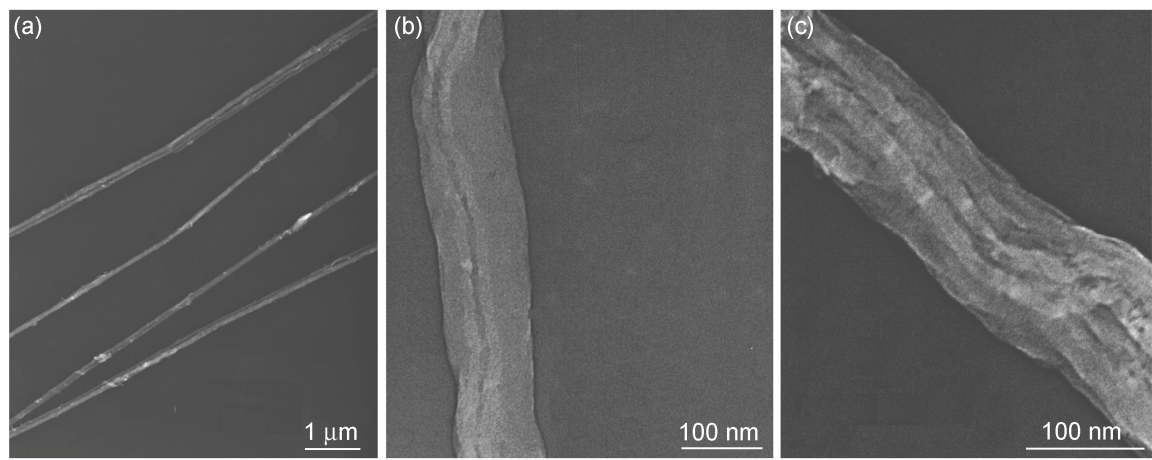

图 6 不同放大倍率的沿轴线嵌入纳米纤维的 MWCNTs 透射电子显微镜图 ${ }^{[43]}$

温下转化为金属 ${ }^{[47] ;}$; (3)金属化合物溶液，如硝酸银 溶液, 可在高温下转化成银 $\left(>400^{\circ} \mathrm{C}\right)^{[48]}$.

Park 等人 ${ }^{[49]}$ 通过多羟基化合工艺合成 40 50 $\mathrm{nm}$ 铜纳米粒子, 开发了具良好分散性、低黏度的铜 粒子导电墨液，其喷印图案显示出类似块体金属的 形貌. Woo 等人 ${ }^{[50]}$ 在铜墨液中加人平均粒径为 $20 \mathrm{~nm}$ 的银纳米粒子, 有效地填充在平均粒径为 $65 \mathrm{~nm}$ 的铜 纳米粒子间的空隙, 明显改善颗粒致密度. 当铜与银 比例为 3:1 时, 颗粒致密度高达 $86 \%$, 可获得比纯铜
金属薄膜更好的导电性, 并可在较低温度 $\left(175 \sim 210^{\circ} \mathrm{C}\right)$ 下将导电图案直写到透明塑料基板(如聚醚)上.

银纳米粒子因其独特的性质(如化学稳定性、优 良催化活性和导电性), 在催化剂、透明导电涂料、导 电墨液等领域得到了广泛应用. 用于喷印的银纳米 粒子墨液须满足下列条件 ${ }^{[51]}$ : 银纳米粒子简单低成 本制备、在溶剂中金属粒子具有良好的分散稳定性, 以及打印的图案具有良好的导电性. 导电银墨液表 现为近似牛顿流变行为, 具有优良的分散稳定性 ${ }^{[52]}$, 
在 $100 \mathrm{~s}^{-1}$ 剪切速率下黏度约为 $3 \mathrm{mPa} \cdot \mathrm{s}$, 表面张力为 30 40 mN/m. 银纳米粒子导电墨液的烧结温度随粒 径减小而单调降低, 这对实现柔性电子的高导电性 至关重要. 将含有约 $20 \mathrm{~nm}$ 直径的纳米银粒子导电墨 液喷印形成的图案在 $200^{\circ} \mathrm{C}$ 下热处理 $30 \mathrm{~min}$ 后, 显示 出类似金属的形貌和导电性.

利用化学还原法和稳定剂(如嗍氢化钾/钠、柠檬 酸钠、抗坏血酸维生素 $\mathrm{C}$, 碳氢化合物和气态氢等) 制备纳米银微粒时, 通常需要采用保护剂, 如聚 (N乙烯基吡咯烷酮)和硫代水杨酸等 ${ }^{[33,54]}$. 然而, 热处 理过程中由于保护剂分解形成的气孔将降低银薄膜 导电性. 将含有还原剂(或银盐)的墨液喷射到含有银 盐(或还原剂)的基板上, 通过加热基板形成导电图案, 可有效避免上述问题 ${ }^{[53]}$.

$\mathrm{Wu}$ 等人 ${ }^{[55]}$ 用乙二醇蒸气凝聚方法直接喷印硝 酸银溶液制备出银导线，低温下硝酸银前体在乙二 醇蒸气中逐渐减少形成银. 图 7 中银阵列图案是通过 喷印不同浓度硝酸银水溶液方法制备, 并在 $250^{\circ} \mathrm{C}$ 的 乙烯乙二醇水蒸气中处理 $10 \mathrm{~min}$. 分析表明, 硝酸银 已完全转换为银, 产生了连续银导线, 电阻率为 $7.314 \times 10^{-5} \Omega \cdot \mathrm{cm}$, 接近块体银的电阻率. Lee 等人 ${ }^{[56]}$ 探讨了电喷涂银纳米胶体制备含银纳米粒子二维功 能结构的工艺方法. 喷嘴喷射一次可获得 100 300 $\mathrm{nm}$ 厚的图案, 打印线圈的电感为 $9.45 \mu \mathrm{H}$, 烧结后电 阻率约为块体银电阻率的 5 倍 $(9.5 \mu \Omega \cdot \mathrm{cm})$. 可通过 EHD 喷印将银纳米粒子打印到电路板上形成导线 ${ }^{[57]}$. 烧结后银线的电阻率 $(4.8 \mu \Omega \cdot \mathrm{cm})$ 是块体银电阻率的 3 倍多(银线的特性阻抗 $18 \Omega$ ).

\section{3 有机喷印材料}

通过精心设计和操纵种类繁多的碳基结构, 有 机/高分子材料可制备出导体、半导体和绝缘体 ${ }^{[33]}$, 兼有聚合物的物理化学性质和金属的电学特性 ${ }^{[58]}$. 通过高分辨率喷印技术可制备出全聚合物薄膜晶体
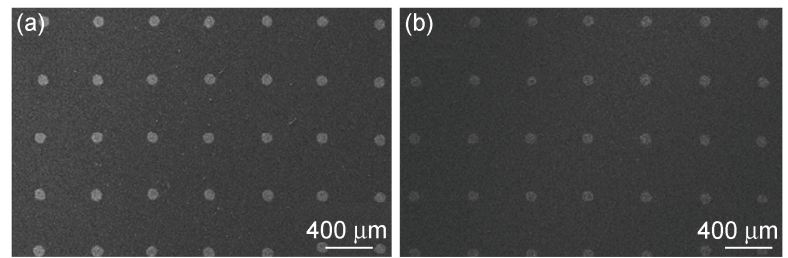

图 7 银阵列形貌图 (a) $5 \mathrm{~mol} / \mathrm{L}$; (b) $14 \mathrm{~mol} / \mathrm{L}^{[55]}$
管, 如图 8 所示 ${ }^{[29]}$.

(i ) 有机绝缘材料. 喷印有机介电薄膜的研究 主要集中在可图案化的栅电介质, 如聚乙烯基苯酚、 聚酰亚胺、丙烯基聚合物和溶胶/凝胶状硅氧烷基混 合聚合物等. 并五苯是应用最广泛的小分子半导体, 易制备出超过 $1 \mathrm{~cm}^{2} / \mathrm{V} \cdot \mathrm{s}$ 的空穴迁移率的薄膜晶体管. 聚乙烯吡咯烷酮(PVP)是一种无定形聚合物，可作为 低温溶液化处理的电介质材料. PVP 含有刚性吡咯烷 酮团, 具有较高的玻璃转化温度 $T_{\mathrm{g}}\left(86^{\circ} \mathrm{C}\right)$, 可与许多 无机盐形成复合物 ${ }^{[39,59]}$. 用电介测量法对掺杂硫氰 酸铵 (NH4SCN)的 PVP 介电行为进行测试后表明: 在 PVP 加人含有导电元素 (如银和钾的盐)对聚合物的 导电性产生显著影响 ${ }^{[40,41]}$. Choi 等人 ${ }^{[60]}$ 用 PVP 电介 质取代 $\mathrm{SiO}_{2}$ 作为柾介电层制备无机氧化锌场效应晶 体管(ZnO FET), 研究表明 PVP 聚合物电介质具有理 想的电性能(高迁移率和高开电流).

(ii) 有机导电材料. 常用的导电聚合物包括聚 吡咯、聚(3; 4-乙撑二氧噻吩)(PEDOT)等. PEDOT 具 有良好的导电性 $(400 \sim 600 \mathrm{~S} / \mathrm{cm})$ 、电化学稳定、光电 特性(高透明)和热稳定性 $\left(230^{\circ} \mathrm{C}\right.$ ) 等, 已得到大量研 究. PEDOT 不溶于普通溶剂, 无法直接在喷印中应 用 ${ }^{[58]}$. 如图 9 所示, 采用水溶性聚合物电解质(如聚苯

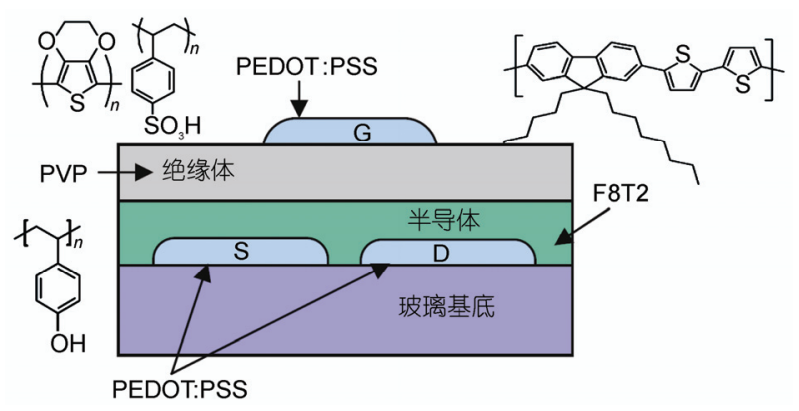

图 8 喷印全聚合物薄膜晶体管示意图 $\mathrm{S}$, 源极; $\mathrm{D}$, 漏极; $\mathrm{G}$, 门极 ${ }^{[29]}$

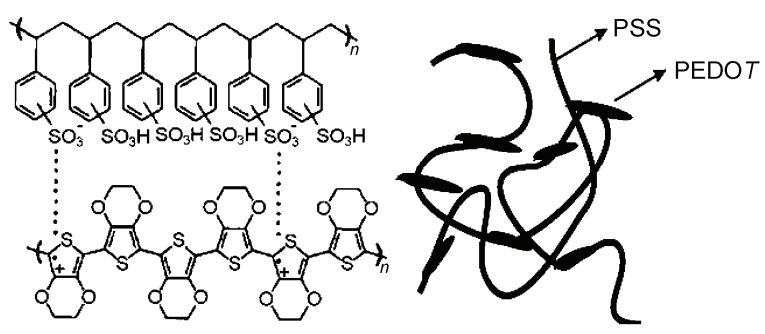

图 9 PEDOT:PSS 的分子结构和 PEDOT 掺杂 PSS 的图式 结构 
乙烯磺酸钠, PSS) 作为电荷平衡反离子掺杂聚合物来 改善 PEDOT 的可溶性, 可得到 PEDOT: PSS ${ }^{[61]}$.

PEDOT:PSS 为深蓝色, 具有 $P$ 型掺杂形式下电 化学稳定、适度透明、高导电性 $(1 \sim 10 \mathrm{~S} / \mathrm{cm})$ 等特性. PEDOT:PSS 虽然具有较透明导体铟锡氧化物(ITO)高 的表面电阻, 但仍可能取代 ITO. 作为胶体分散剂, PEDOT:PSS 可稳定存在于水中, 可用多种方法形成 薄膜 ${ }^{[62]}$. Ballarin 等人 ${ }^{[63]}$ 比较了用喷印和旋涂方法形 成的连续 PEDOT: PSS 薄膜, 发现二者表面和电化学 性能无明显差异, 但用喷印方法更高效、更洁净.
Eom 等人 ${ }^{[64]}$ 喷印出 PEDOT:PSS 层, 测试表明这种新 型的 PEDOT:PSS 墨液可以提高光电性能, 这主要得 益于打印头在甘油和 EGBE 表面活性剂的作用下具 有良好的润湿、扩散和干燥特性. 图 10 是不添加或添 加不同浓度表面剂喷印的 PEDOT:PSS 薄膜表面轮廓 图. 图 11 是喷印 PEDOT:PSS 薄膜的导电性测量结果, 分别为 $0.782,152,164$ 和 $1.28 \mathrm{~S} / \mathrm{cm}$. 图 12 是添加不 同浓度表面剂喷印的 PEDOT:PSS 层太阳能电池在黑 暗状态下模拟 AM1.5 照度为 $100 \mathrm{~mW} / \mathrm{cm}^{2}$ 的电流密 度与电压关系图 $(J-V)$. Hohnholz 等人 ${ }^{[65]}$ 研究表明,
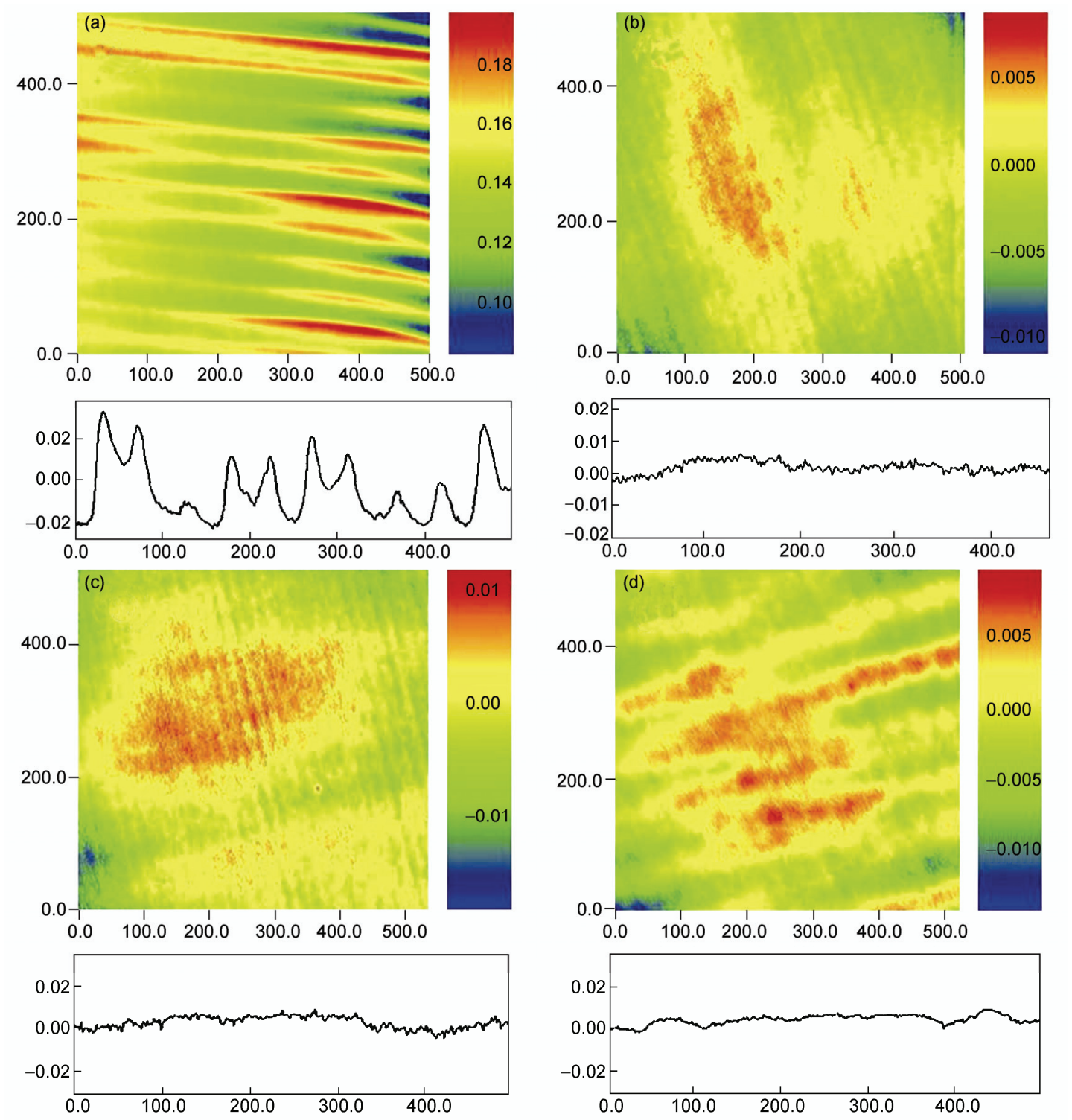

图 10 喷印的 PEDOT: PSS 膜表面照片

(a) 无添加剂(纯 PEDOT:PSS); (b) $6 \%$ 甘油; (c) $6 \%$ 甘油和 $0.2 \% \mathrm{EGBE;} \mathrm{(d)} 6 \%$ 甘油和 $0.4 \% \mathrm{EGBE}^{[64]}$ 


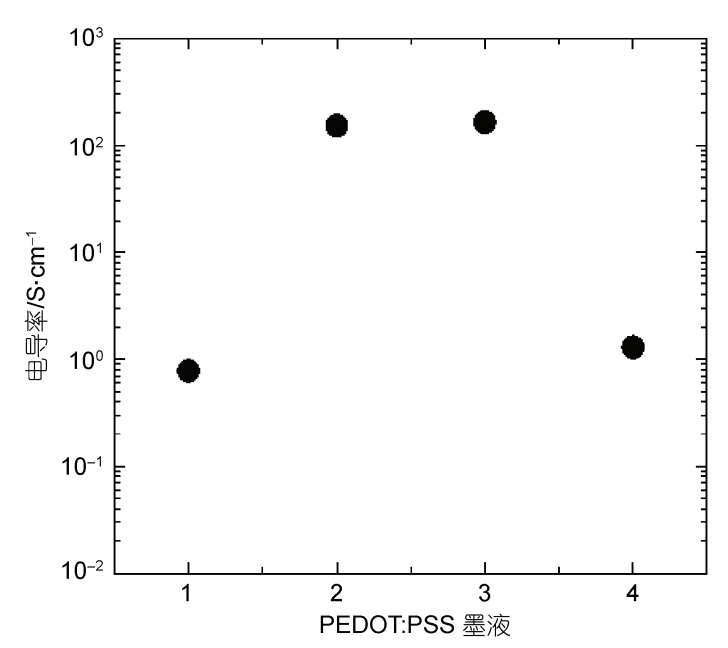

图 11 喷墨打印 PEDOT:PSS 的电导率

(1) 无添加剂; (2) 6\%甘油; (3) 6\%甘油和 $0.2 \% \mathrm{EGBE;} \mathrm{(4)} 6 \%$ 甘油和 $0.4 \mathrm{EGBE}^{[64]}$

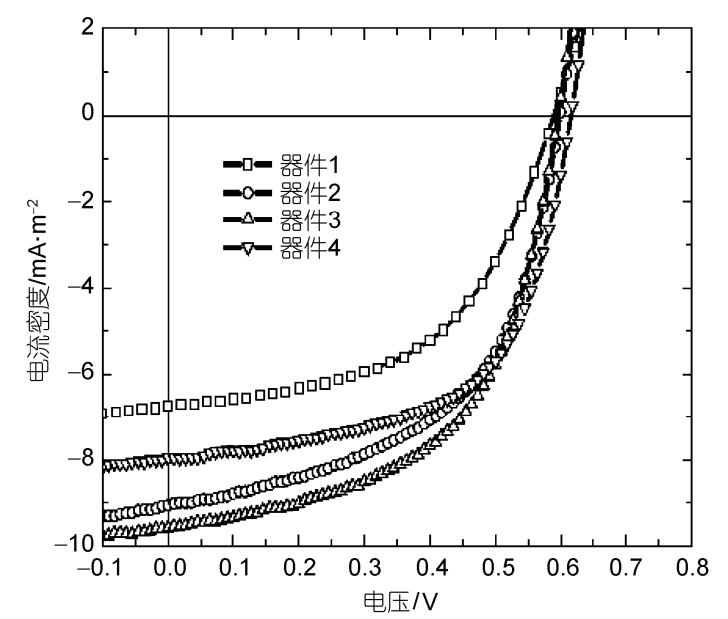

图 12 太阳能电池的电流密度与电压关系 $(\boldsymbol{J}-\boldsymbol{V}$ 特性)图 (1) 无添加剂; (2) $6 \%$ 甘油; (3) $6 \%$ 甘油和 $0.2 \% \mathrm{EGBE;} \mathrm{(4)} 6 \%$ 甘油和 $0.4 \mathrm{EGBE}^{[64]}$

外加溶剂对改善 PEDOT:PSS 薄膜导电性的效果并不 明显, 随着薄膜厚度的增加, 整体导电性取决于 PEDOT 本身. Kwon 等人 ${ }^{[6]]}$ 认为电阻降低源于 PEDOT: PSS 膜厚度的增加, 因为薄膜厚度的增加使得载流路 径增多.

\section{4 混合/纳米复合材料}

聚合物和碳纳米管 $(\mathrm{P} / \mathrm{CNTs})$ 复合材料具有良好 的力、热、光、电等性能, 可与绝缘、铁电和导电聚 合物复合, 在纳米器件中应用前景广泛. Jeong 等人 ${ }^{[67]}$
配置了基于热交联型有机硅氧烷基有机-无机混合溶 胶/凝胶材料的功能墨液, 非常适合喷印, 制备的介 电薄膜具有良好的表面形貌, 粗鋉度只有 $0.3 \mathrm{~nm}$, 即 使在 $90 \mathrm{~V}$ 偏压作用下, 介电层的泄漏电流仍小于 $10^{-6} \mathrm{~A} / \mathrm{cm}^{2}$, 介电常数为 4.9. 实验证实喷印介电层拥 有与旋涂介电层类似的性能, 但对于喷印介电层的 有机晶体管, 残余溶剂分子会引起较大的迟滞行为 和阈值电压增大的现象.

分散度直接影响纳米颗粒溶液的流变和触变特 性, 继而影响沉积薄膜质量 ${ }^{[68]}$. 利用三辊轧机研磨 进行材料混合效果良好，可有效分散溶剂中大块银 纳米粒子团聚物 ${ }^{[69]}$, 并可得到最小电阻率的薄膜 (约 $3.64 \times 10^{2} \mu \Omega \cdot \mathrm{cm}$ ). 用水作为分散剂具有诸多优势, 如降低挥发性有机化合物的含量具有良好的环保特 性、不易燃烧、低温下完全蒸发等.

\section{3 喷印工艺}

喷印技术早已应用于多孔表面打印文本和图像、 复杂三维结构的快速原型等. 最近, 喷印技术用于功 能材料直写电子器件 ${ }^{[26,70,71]}$, 但面临墨液配制、驱动 模式、基板选择和溶剂挥发性控制等挑战. 传统的 “推” 模式喷印技术已得到较为广泛的研究与应用, EHD 等“拉”模式的高分辨率打印技术在器件制造应 用研究才刚刚开始.

\section{1 传统喷印技术}

(i ) 驱动形式. 传统喷印 ${ }^{[71]}$ 主要有两种液滴生 成方式: 连续喷印 $(\mathrm{CIJ})$ 一一由液滴构成的液柱连续 从喷嘴喷出, 通过加载在液柱上的周期扰动产生间 隔和大小均匀的液滴; 按需喷印(DOD)——液滴按需 要喷出. 喷印过程最重要因素是墨液的表面张力和 黏性, 以及驱动的频率和幅度. 与按需喷印相比, 连 续喷印更适用于低黏度流体, 滴落速度高, 需要墨液 量大. 按需喷印定位精度高、可控性好、节约材料, 被 广泛应用.

按需喷印使用脉冲推动液滴, 驱动方式包括热 泡法和压电法 ${ }^{[71]}$ (如图 13 所示), 以及热屈曲法 ${ }^{[30]}$ 和 声激励法 ${ }^{[72]}$ 等. 如图 13(a)所示, 热泡法中液体薄膜 通过加热器局部加热, 数微秒内蒸汽泡沫迅速扩大, 喷出墨滴. 初始驱动压力接近过热极限条件下液体 饱和蒸气压(通常水约为 $4 \mathrm{MPa}$, 有机溶剂约为 $1 \mathrm{MPa}$ ). 热泡法打印最小液滴约为 $0.5 \mu \mathrm{m}$ (水)、 $1 \mu \mathrm{m}$ (甲醇和 
甲苯 $)^{[30]}$. 当温度提高到 $300^{\circ} \mathrm{C}$ 时易导致喷嘴堵塞 ${ }^{[27]}$, 不适合打印熔融聚合物 ${ }^{[37]}$. 如图 13(b)所示, 压电法 中依靠压电膜的机械作用产生脉冲 ${ }^{[37]}$, 通常喷嘴尺

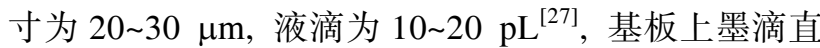
径约为滴落过程墨滴直径的 2 倍.

常通过制造大量密集的喷墨头阵列实现高效打 印，如打印头包含几十、几百甚至几千个独立喷嘴, 墨液由同一个导管供给, 但是每个喷头可单独进行 控制 ${ }^{[70]}$. 使用块体压电打印头可取得足够的驱动力, 但导致阵列尺寸过大或打印头数目受到限制 ${ }^{[21]}$. 由 于蒸汽泡的自由膨胀, 热泡打印仅需一个加热器进 行驱动, 可通过微制造技术加工出大面积喷头阵 列 ${ }^{[30]}$. Wang 等人 ${ }^{[73]}$ 开发了热泡喷头密集阵列, 可产 生直径为 $3 \mu \mathrm{m}$ 的水滴.

(ii) 喷印机理. 为实现高效、高分辨率和高可 靠打印, 需要研究精确操控掺杂大量固体粒子、非牛 顿液体射流或液滴的复杂物理过程. 按需喷墨中, 液 滴形成的主要阶段包括: 喷射和射流拉伸、喷嘴液体 飞线、液体飞线收缩、主/卫星液滴形成, 以及主/卫 星液滴重新结合等 ${ }^{[74]}$. 为精确控制射流形成以及喷 射运动, 需要对高剪切速率的材料性质、射流不稳定 性、液滴成形与运动、液带的拉伸, 以及飞行过程中 射流和液滴的气动性和静电作用进行理论、仿真和实 验研究 ${ }^{[75]}$.

瞬态液体速度分布引起喷嘴处液体表面变形形 成颈缩, 颈缩加剧就会断裂成小液滴. 控制液体射流 和滴落行为的主要作用力包括惯性力、黏性力和表面 张力. 当液滴喷出, 能量转化成黏性流动、液滴表面 张力和动能. 除主液滴外, 紧随其后还会产生更细小 的卫星液滴, 并随后可能与主液滴再结合, 其行为无 法通过线性 Rayleigh 分析方法进行预测 ${ }^{[36]}$. 卫星液 滴的抑制非常关键 ${ }^{[37]}$, 通常可通过改变驱动扰动的
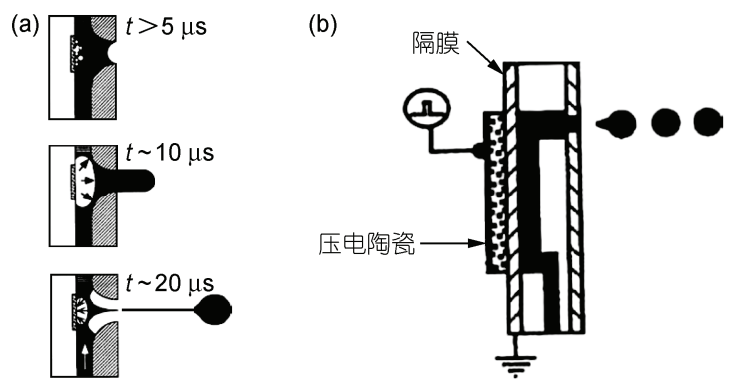

图 13 喷墨打印的喷头结构

(a) 热泡式; (b) 压电式 ${ }^{[71]}$
幅度来控制卫星液滴生成.

通常，喷嘴直径越小可获得越小的液滴和越高 的分辨率. 然而, 当喷嘴尺寸降低, 表面张力和黏性 力将逐渐增加, 需要更大的驱动力对液体进行加速. 墨液配方需解决 “第一滴墨滴生成” 问题, 避免墨液 部分干燥导致喷嘴堵塞 ${ }^{[27]}$. 主要解决手段包括：(1) 对喷嘴进行清洗和盖帽; (2) 采用低挥发性墨液和吸 收性基板; (3) 通过加热、干燥或紫外等固化墨液 ${ }^{[36]}$ 目前，喷头、墨液和基板的物理化学相容性机理尚不 清楚.

（iii）性能控制. 近年来，随着活性有机材料技 术发展以及半导体薄膜打印精度的提高, 喷印 OTFT 性能得到较大提升. 可喷印的几何图案包括岛 (电 极)、线(互联结构)、面(大面积的层结构). 在液滴层 叠形成液态线的打印过程中，由于液态线的扩散和干 燥等将导致颈缩现象等不稳定性问题 ${ }^{[76]}$, 使长距离 液态线的特征尺寸和形貌的精确控制极其困难. TFT 的电荷载流子传输性能取决于其形貌, 为避免器件的 短路和断路, 须通过形貌控制实现无针孔层的喷印。 形貌控制主要通过调节沉积条件实现，如基板表面改 性、溶剂属性调整、采用分子间作用较强的 P-P 型有 机半导体材料等 ${ }^{[77]}$. 低黏度流体不会在喷嘴发生堵 塞，可生成一致的液滴，并通过液体蒸发实现固化， 其液滴尺寸控制取决于液滴在基板上的扩散行为 ${ }^{[78]}$.

溶液扩散可通过光刻或其他方法辅助在基板表 面不同区域采用不同润湿性材料 ${ }^{[79]}$ 或拓扑结构 ${ }^{[80]}$ 等 进行控制, 如基于润湿性差异的自对准打印方法 ${ }^{[81]}$. 图 14 所示为通过上述策略喷印出的沟道长度为微米 尺度的全聚合物 $\mathrm{TFT}^{[29]}$ : (a)是在预处理表面上进行 高分辨率喷印的示意图; (b)是喷印 PEDOT: PSS 源极 和漏极的原子力显微镜图, 其中源漏极通过 $5 \mu \mathrm{m}$ 宽 的聚酰亚胺进行分离; (c)是 F8T2 半导体层, 以及 PEDOT:PSS 源极和漏极; (d) 是器件的光学显微图. 通过对喷印过程进行适当的控制, $\mathrm{Bao}^{[82]}$ 制造出沟道 尺寸为 $250 \mathrm{~nm}$ 的晶体管. Sirringhaus 等人 ${ }^{[83]}$ 利用传统 喷印设备在无光刻辅助的情况下通过自对准打印技 术获得小于 $100 \mathrm{~nm}$ 的线宽. 通过喷墨和激光烧蚀/烧结 的组合方法在聚合物层上制造出无源和有源元件 ${ }^{[84]}$.

打印过程中液滴沉积到基板上的定位精度控制 十分重要。喷出的液滴经过特定的飞行轨迹后着落 在基板上，定位精度可控制在 $5 \sim 10 \mu \mathrm{m}^{[1]}$. 液滴的定 位精度不仅取决于喷头的定位精度，也取决于喷头 


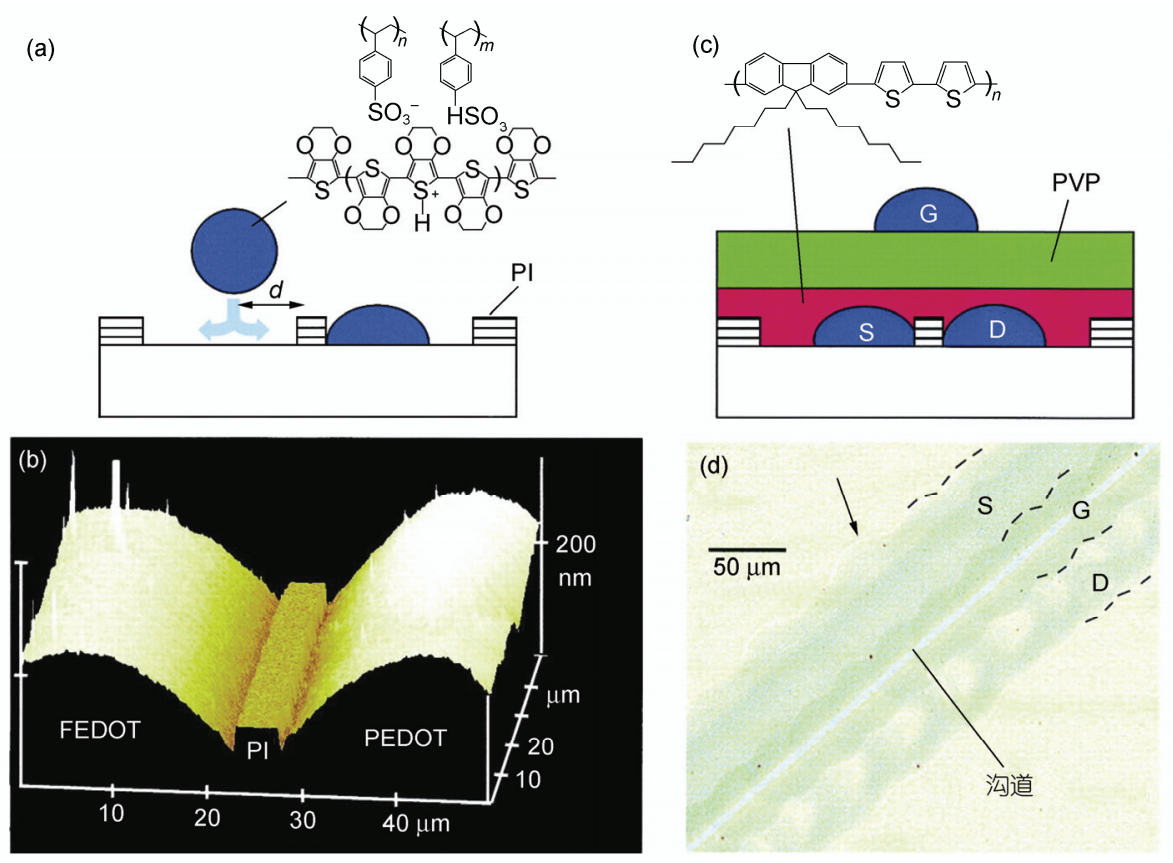

图 14 自对准喷印制造的 TFT 示意图

(a) 透明基板上实现高分辨率喷墨打印示意图; (b) 喷墨打印的 PEDOT:PSS 源极和漏极被长为 $5 \mu \mathrm{m}$ 聚酰亚胺线分开的原子力显微图; (c) 喷墨 打印半导体材料 F8T2 为棚极的 TFT 示意图; (d) 喷墨打印的 TFT $(L=5 \mu \mathrm{m})$ 的光学图片. S, 源极; D, 漏极; G, 门极

与基板的角度, 较强的侧风也会使液滴偏斜. 通过减 小喷嘴与基板间的距离可提高定位精度. 另外, 保持 喷嘴处液滴的一致性润湿非常重要, 否则液滴将偏 斜一定角度 ${ }^{[37]}$.

\section{2 电流体动力打印}

与传统喷印采用“推”方式不同, EHD 喷印采用电 场驱动以“拉”方式从液雉顶端产生极细的射流，分 别形成喷雾(电喷涂, 如图 19 所示)、纤维(电纺丝, 如 图 21 所示)和液滴(电喷印, 如图 24 所示). EHD 喷印 具有许多独特的优势, 如可实现亚微米分辨率 ${ }^{[85]}$ 、颗 粒或聚合物溶液易于喷出而不发生堵塞等. EHD 喷 印分辨率不受喷嘴直径的直接影响 ${ }^{[78]}$, 可采用较粗 的喷嘴在避免溶液堵塞喷嘴的前提下获得亚微米的 液滴 ${ }^{[86]}$. EHD 喷印(电喷涂、电纺丝和电喷印)非常适 合于复杂和高精度图案化, 在柔性电子制造中具有 广泛的应用前景, 如电喷涂、电纺丝、电喷印可分别 用于制备柔性电子的薄膜层、器件互联、电极.

(i ) 打印机理. EHD 喷印技术均应用电流体 动力学机理和类似的实验装置 ${ }^{[78,87]}$, 其喷印过程如 图 15 所示: 利用电场将液体从喷嘴口拉出形成泰勒雉, 由于喷嘴具有较高的电势, 喷嘴处的液体会受到电致
切应力的作用; 当局部电荷力超过液体表面张力后, 带电液体从喷嘴处喷射, 然后破裂成液柱或小 液滴 ${ }^{[88}$. 通过改变流速、电压、液体性质和喷嘴结构, 可形成具有不同射流形状和破碎机理的 EHD 喷印 模式 ${ }^{[89]}$.

EHD 喷印可采用连续雉射流 ${ }^{[56]}$ 和脉冲喷印 ${ }^{[90]}$ 模 式实现图案化. 连续雉射流采用直流电压形成连续

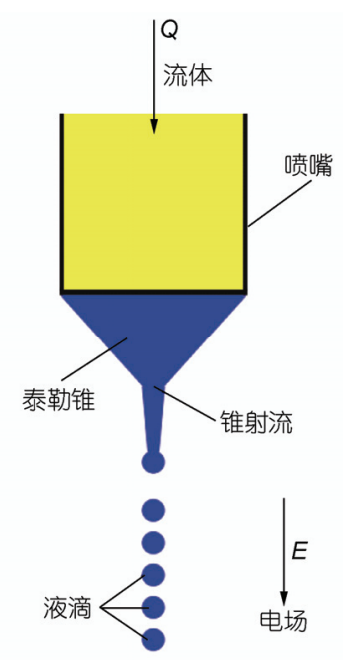

图 15 通过电流体尖端液流产生液滴 ${ }^{[8]}$ 
的线，脉冲喷印采用脉冲电压 ${ }^{[11]}$ 或较低的直流电压 以按需打印方式形成线或一系列的点. 随着脉冲电 压频率的升高, 液雉不断震荡或破碎成许多小液滴, 无法保证在每个脉冲作用下均发生滴落 ${ }^{[87]}$. 如液体 从喷嘴流出时仍保持非常高的电势, 在电场力作用 下将生成更小的液滴.

EHD 喷印装置通常由墨液盒或精密流量洜、镀 有金属的喷嘴、高压发生器和收集电极等组成 ${ }^{[78,92]}$. 不同 EHD 喷印方式的区别主要在于溶液性质以及针 头与电极间的电压和距离. EHD 喷印装置包括两个 电极: 通常采用不锈钢针头作为第一个电极; 第二 个电极采用针、环或平面等结构, 直接位于针头(喷 嘴)的下方并接地. 通过控制注射原的活塞运动, 溶 胶/凝胶溶液在两电极间的电压作用下喷射出液滴或 纤维. 如图 16 所示, 当液滴处于高电势差空间中, 其 形状稳定性由重力 $F_{\mathrm{g}}$ 、表面张力 $F_{\mathrm{st}}$ 和电场力 $F_{\mathrm{e}}$ 共同 确定 ${ }^{[93]}$. 如图 17 所示, 如果电势差不高, 液滴只受到 有限静电拉力; 当液滴表面继续变形, 电场引起电荷 集中并最终形成泰勒雉; 当达到临界电压, 电场力与 表面张力平衡被打破, 高速射流从锥尖喷射出来 ${ }^{[94]}$. 针对不同溶液进行相应的参数调整, 可实现电喷涂、电
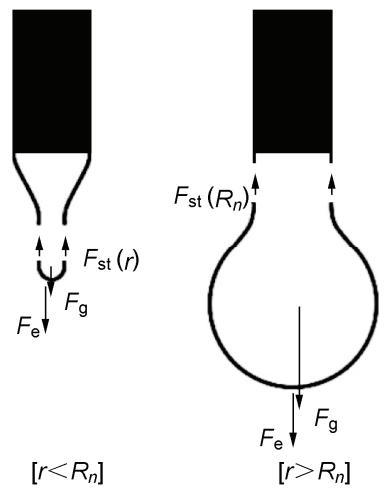

图 16 在喷嘴顶端的液体半月雉示意图 ${ }^{[93]}$

纺丝和电喷印等 3 种 EHD 喷印方法(见表 3).

如图 18 所示 ${ }^{[86],}$ 可通过改变作用电压和流速实 现垂滴、微垂滴和雉射流等喷射模式. 在垂滴模式下, 无电场作用的液滴在喷嘴处膨胀后形成连续液滴, 此时液体在喷嘴口顶端形成锥形而非球形弯月面. 在微垂滴模式下，单分散相液滴直接在半月雉顶部 形成 ${ }^{[93]}$. 在雉射流模式下, 锥体被拉长产生射流, 然 后破碎成小液滴。尚未见到脉冲雉射流模式中有关 液滴发生频率，以及控制液滴尺寸和空间位置的研
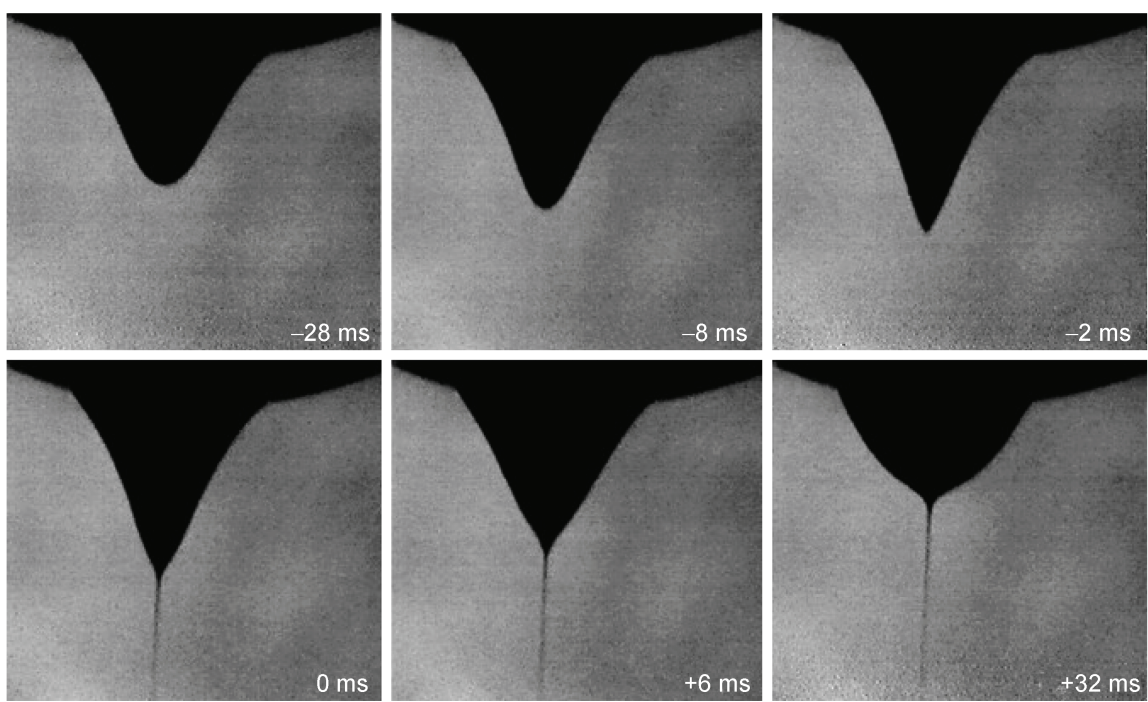

图 17 液体的形状演化 ${ }^{[94]}$

表 3 电流体动力打印方法比较

\begin{tabular}{clcccc}
\hline EHD 喷印 & 打印模式 & 电压 $/ \mathrm{kV}$ & 电极距离 $/ \mathrm{mm}$ & 溶液 & 图案 \\
\hline 电喷印 & 锥射流, 微垂滴 & $0.5 \sim 3$ & $0.1 \sim 1$ & 聚合物, 纳米粒子 & 点和离散线 \\
电纺丝 & 锥射流, 多射流 & $1 \sim 10$ & $10 \sim 50$ & 聚合物, 纳米粒子 & 纤维(连续线) \\
电喷涂 & 锥射流, 多射流 & $15 \sim 30$ & $100 \sim 250$ & 各种材料 & 溥膜 \\
\hline
\end{tabular}




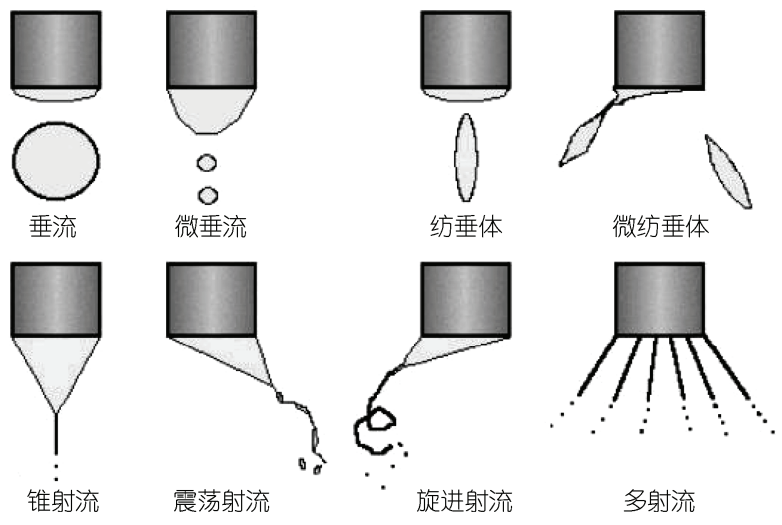

图 18 电流体动力喷射模式 ${ }^{[86]}$

究报道 ${ }^{[87]}$. 为预测静电力作用下喷嘴喷出的液滴半 径 $r$, 建立以下力平衡方程 ${ }^{[93]}$ :

$$
F_{\mathrm{g}}-F_{\mathrm{st}}+F_{\mathrm{e}}=0 .
$$

通过试错方法可计算出三力平衡时的半径 $r$. 结果 表明: $r$ 是 3 个力的函数, 同时 3 个力也受 $r$ 的影响 ${ }^{[6,93]}$.

(ii) 电喷涂. 如图 19 所示, 电喷涂(电雾化)用 电场将液滴雾化, 通常采用含有纳米粒子的溶胶/凝 胶溶液, 其黏度往往高于传统喷印的墨液. 电喷涂可 用于处理特殊溶液, 如沉积 PZT 薄/厚膜 ${ }^{[95]}$ 、利用溶 胶/ 凝胶溶液前驱物沉积金属 ${ }^{[78]}$ 等. 电喷涂具有以下 优势:（1）生成的液滴尺寸极小(可达 $10 \mathrm{~nm}$ ); (2) 可 通过调节流速和电压控制液滴的尺寸和电荷; (3) 液 滴尺寸分布近似于均匀分散 ${ }^{[86,96]}$. 电喷涂中相同尺 寸的粒子具有相似的热动力学状态, 可得到较其他 方法更均匀的薄膜, 从而减少柔性电子中空隙和裂 缝的数量 ${ }^{[86]}$. 电喷涂已用于生产有机薄膜(目前实现 薄膜为 $15 \mathrm{~nm}^{[97]}$ ), 也可作为直写技术 (如图 20 所 示 $^{[86]}$. 目前电喷涂研究主要包括纳米电喷涂 ${ }^{[88]}$ 、喷 雾成型 ${ }^{[99]}$ 、薄膜沉积 ${ }^{[96]}$ 、电流动力学机理 ${ }^{[100]}$ 等方面.

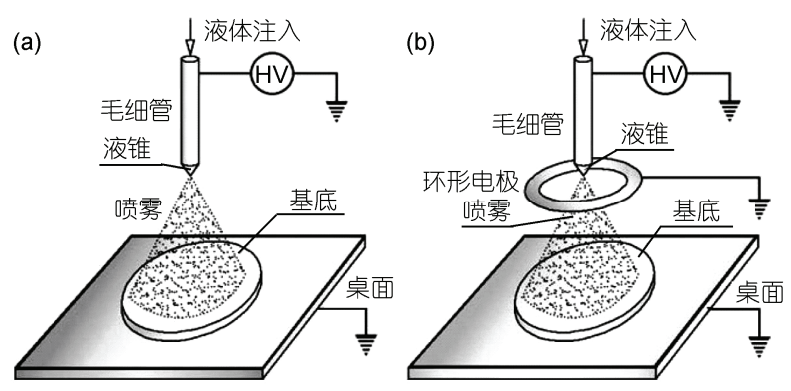

图 19 电喷涂喷头示意图 ${ }^{[96]}$

(a) 普通喷嘴; (b) $X$ 向牵引系统 (a)
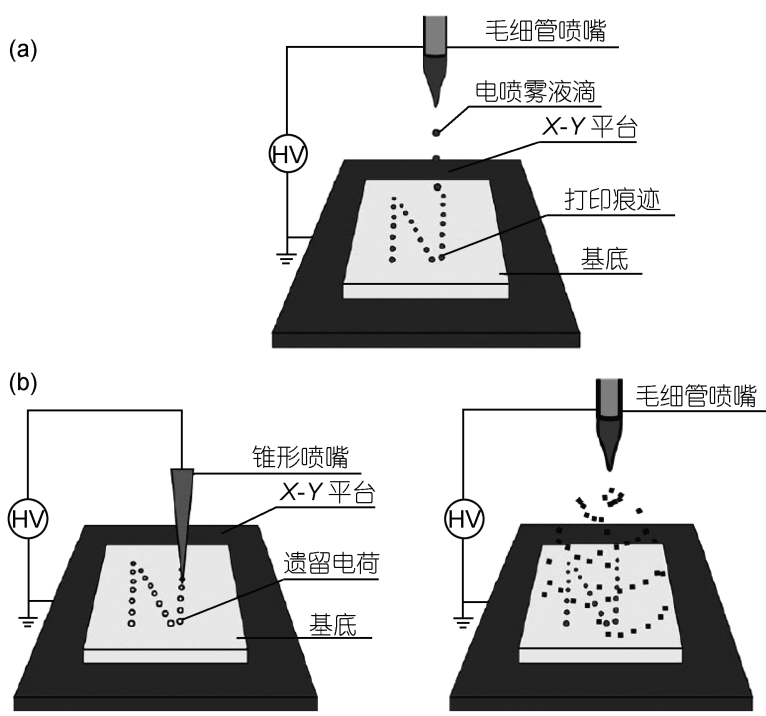

图 20 电喷涂直写技术

(a) 采用电喷涂液滴的一步工艺; (b) 两步工艺: 先用尖针头进行离 子沉积, 然后进行纳米电喷涂 ${ }^{[86]}$

带电液滴的喷雾沉积效率可通过电场进行控制, 通常高于不带电液滴 ${ }^{[66,96]}$. 如图 19 所示 ${ }^{[96]}$, 电喷涂 通常采用直接喷涂喷嘴和萃取喷嘴两种模式，两者 主要区别在于毛细管和基板之间增加一个环形电极, 以避免由于基板电极损伤造成薄膜的不均性，但是 部分液滴会落在环形电极上. 在图 19(a)中, 液体从 喷嘴处弯月锥顶端直接喷射出液滴碎片, 在分离过 程中形成规则的大液滴、小液滴或拉伸纺锤体. 在图 19(b)中，液体被拉伸成小射流，由于射流的不稳定 最终破碎成液滴. 观察到的射流光滑、平稳, 或沿着 规则的路径运动(围着毛细管旋转或者在其平面内振 荡). Fujihara 等人 ${ }^{[101]}$ 通过电喷涂制备出了用于染料 敏化太阳能电池的大面积 $\mathrm{TiO}_{2}$ 电极层 $\left(20 \mathrm{~cm}^{2}\right)$. 在充 满氮气和乙醇的环境中, Cich 等人 ${ }^{[102]}$ 在硅(111)基板 上电喷涂出了等离子活性显示层. 研究表明, 薄膜的 形貌和荧光强度取决于电压、流速等沉积参数.

（iii）电纺丝. 尽管电纺丝基本思想的提出可追 溯到 20 世纪 30 年代, 但目前对其理解仍然有限 ${ }^{[103]}$. 电 纺丝技术被公认为是制造亚微米乃至纳米纤维的高 效技术之一, 具有相分离、模板法、拉丝、自组装等 纳米纤维制造方法无法謧美的灵活性、柔性和易于纤 维生产等优势 ${ }^{[92,103]}$. 电纺丝过程如图 21 所示 ${ }^{[22]}$, 利用 高压使聚合物溶液/熔液形成带电的射流由喷嘴喷出, 在到达收集电极之前, 射流会干燥或者固化, 最后收 


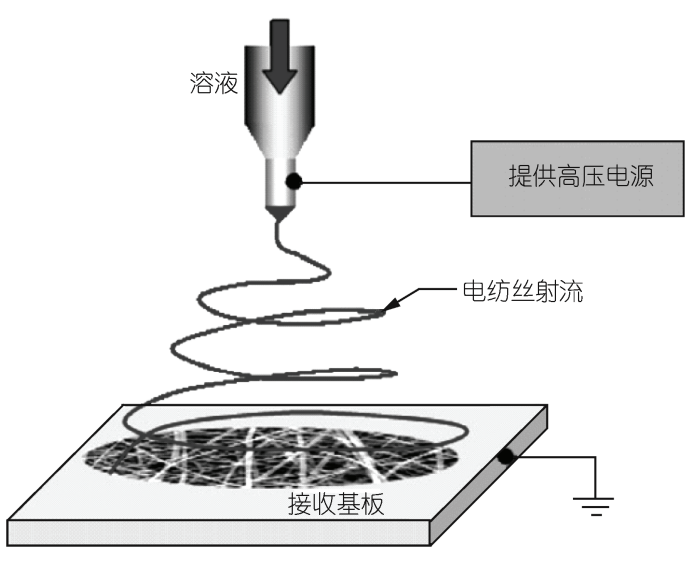

图 21 电纺丝过程 ${ }^{[92]}$

集到由小纤维连成的网状物 ${ }^{[103]}$. 最近发展了无喷丝 头技术 ${ }^{[104]}$ 和近场电纺丝技术 ${ }^{[105]}$ 等新的电纺丝模式. 目前已有 100 多种材料成功地被电纺成极细的纤 维 ${ }^{[103]}$, 其中大多数是聚合物、无机物以及掺杂纳米 材料(纳米管、纳米颗粒)的复合材料. 理论上, 只要给 电纺丝喷嘴供给足够的溶液, 射出的流体就会形成 连续不断的纤维. 最近, 用电纺丝制备的导电聚合物 纳米纤维已用于微纳米器件的设计和构造 ${ }^{[105,106]}$.

在电纺丝中, 虽然在喷丝头顶部射流稳定, 在雉 尖喷出的极细射流加速飞向收集电极同时固化成纤 维过程中, 将出现“鞭动”等复杂的动力学行为. 如图 22 所示 ${ }^{[94]}$, 电纺丝在正常状态下将产生几类不稳定, 因而实现其可控性非常重要. 目前已开展了少量关 于纺丝控制的研究(包括用电场对纤维定向、转动机 械轴收集纤维等 ${ }^{[105]}$, 以及大量关于电纺丝的物理化 学基础研究 ${ }^{[103]}$ (包括聚合物溶液浓度、施加电压和电 极间距离等).

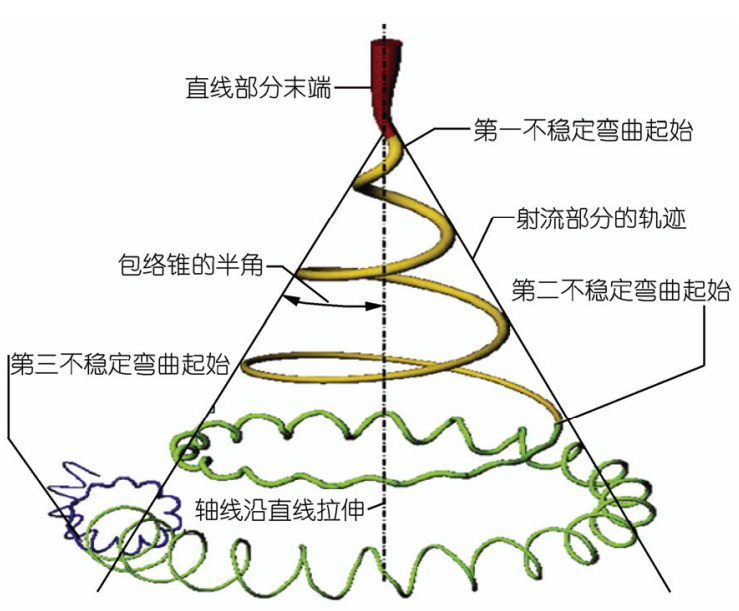

图 22 液滴形状随时间和电压改变的演化过程 ${ }^{[94]}$

MacDiarmid ${ }^{[106]}$ 在其诺贝尔获奖演讲“合成金属: 有机聚合物的新角色” 中预测了电纺丝在柔性电子中 的应用, 并加工出世界上第一根导体聚合物纤维(直 径约为 950 2100 nm). 在此之后, Chronakis 等人 ${ }^{[58]}$ 制 备出直径为 70 300 $\mathrm{nm}$ 的纳米纤维. Jeong 等人 ${ }^{[107]}$ 制 出了多壁碳纳米管和尼龙的复合纳米纤维, 并测试了 其电压-电流的性能. Zhang 等人 ${ }^{[43]}$ 电纺丝出直径约 100 $\mathrm{nm}$ 的纤维, 并在两个平行电极区间内实现定向排列.

最近, Sun 等人 ${ }^{[105]}$ 提出了近场电纺丝技术(NFES), 以一种直接、连续、可控的方式沉积固态纳米纤维(如 图 23(a)所示). 如图 23(b) 所示, NFES 与传统电纺丝 技术的主要不同包括: 两电极间的距离 $h$ 通常为 500 $\mu \mathrm{m} \sim 3 \mathrm{~mm}$; 聚合物溶液以类似蘸笔技术的方式实现 供给; 尖端为直径 $25 \mu \mathrm{m}$ 的铇针尖; 电压因电极间距 减小而降低等. 在图 23(c)中, 从聚合物溶液中拉出 直径为 $50 \mu \mathrm{m}$ 的液滴, 并可观察到泰勒雉(图 23(d)).
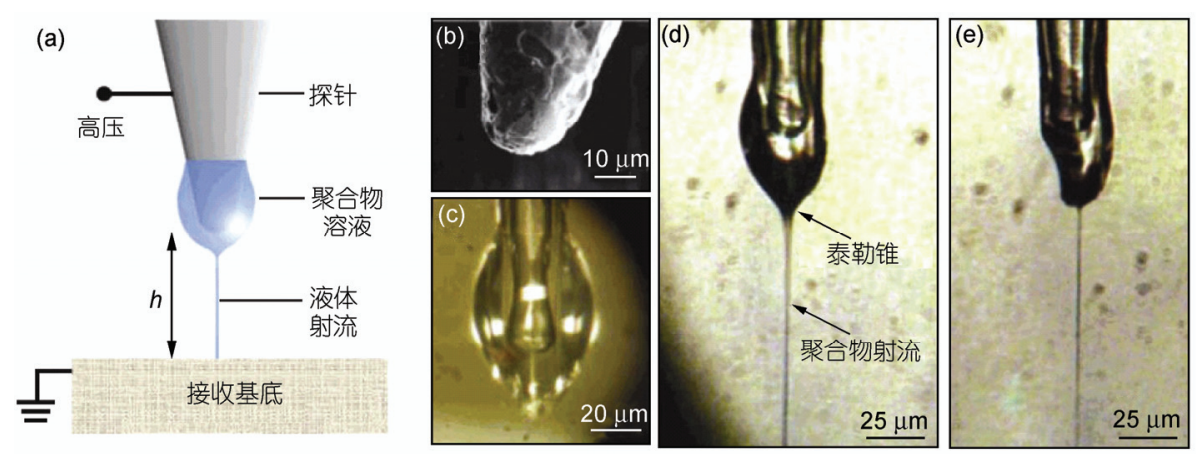

图 23 近场静电纺丝工艺 ${ }^{[105]}$

(a) 近场静电纺丝示意图, 聚合物溶液类似浸笔的方式连接到铇电极尖端; (b) 针头的 SEM 图片; (c) 铇电极尖端直径为 $50 \mu \mathrm{m}$ 的聚合物液滴的 光学图片; (d) 光学显微下, 在电场中聚合物射流从泰勒锥中喷出; (e) 随着聚合物射流的形成, 泰勒雉处液滴的体积减小 
如图 23(e)所示, 随着铇针尖的聚合物溶液不断消耗导 致液滴直径收缩, 可实现更小的泰勒雉和纳米纤维. NFES 技术集成蘸笔、喷印和常规电纺丝技术的特点， 有望实现 $100 \mathrm{~nm}$ 以下纤维的可控制备.

（iv）电喷印。电喷印采用微垂流模式下按需喷 墨控制方式, 可产生非常均匀的液滴并形成任意高 精度图案. 适用于电喷印的材料范围非常广泛, 包括 从绝缘聚合物到导电聚合物, 以及从县浊液到单壁 碳纳米管溶液等. 已有研究大多通过改变偏置电压 和脉冲电压以实现对射流按需打印的操控 ${ }^{[03]}$, 以及 确定喷射所需的最优脉冲条件等, 在电喷印机理方 面的研究较少. 电喷印的喷嘴结构和系统组成如图 24 所示 ${ }^{[85]}$. 电喷印中, 射流从针尖(第一个电极)射出, 液滴在基板(第二个电极)上沉积, 通过移动平台控制 图案形状. 针头的内径通常为 $0.1 \sim 1 \mathrm{~mm}$, 通过制造 更小内径的针头可获得更高的分辨率. 电喷印产生
的液滴至少比针头尺寸小一个数量级 ${ }^{[85]}$, 能达到微 米甚至纳米尺寸.

Park 等人 ${ }^{[85]}$ 研究了静电诱导流体从微毛细管喷 嘴中流出形成射流的动力学行为, 打印出亚微米分 辨率的图案, 可用于制作典型电路和功能晶体管(特 征尺寸高达 $1 \mu \mathrm{m}$ )的金属电极、互联导线和探针点. 可打印出的 $\mathrm{Au}$ 电极线宽为 $2 \mu \mathrm{m}$ (刻蚀和脱模后), 源 极和漏极电极间的沟道距离为 $1 \mu \mathrm{m}$. 如果用内径为 2 $\mu \mathrm{m}$ 的针头以 $10 \mu \mathrm{m} / \mathrm{s}$ 速度打印连续的线图案, 线宽为 3 $\mu \mathrm{m}$; 如果采用内径 $1 \mu \mathrm{m}$ 的针头, 线宽可达到 $700 \mathrm{~nm}$. 发现用单壁碳纳米管作为半导体连接偶电极，可有 效提高薄膜晶体管的性能.

Sekitani 等人 ${ }^{[108]}$ 采用类似装置实现了亚飞升液 滴精度打印，在高迁移率有机半导体表面打印了分 辨率为 $1 \mu \mathrm{m}$ 的金属结点，从而制作出 $\mathrm{P}$ 型和 $\mathrm{N}$ 型沟 道晶体管. 采用自组装单层膜制作晶体管的超薄低 (a)

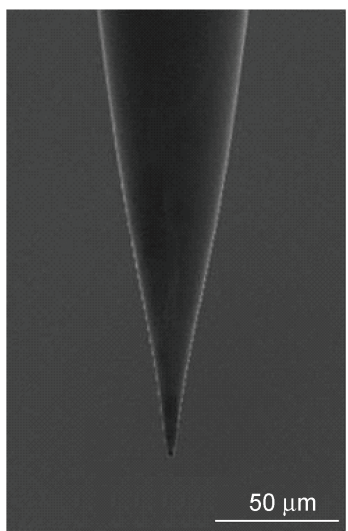

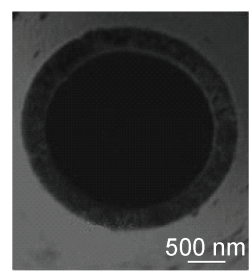

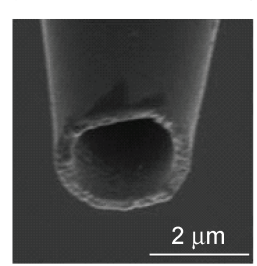

(b)

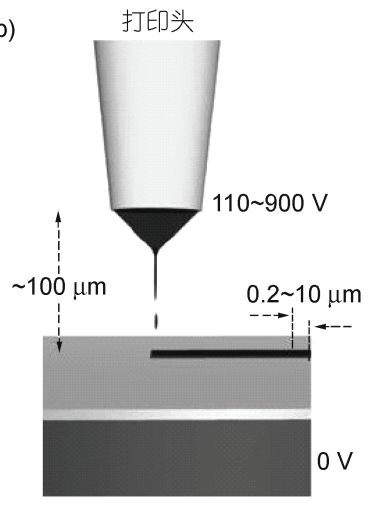

(c)
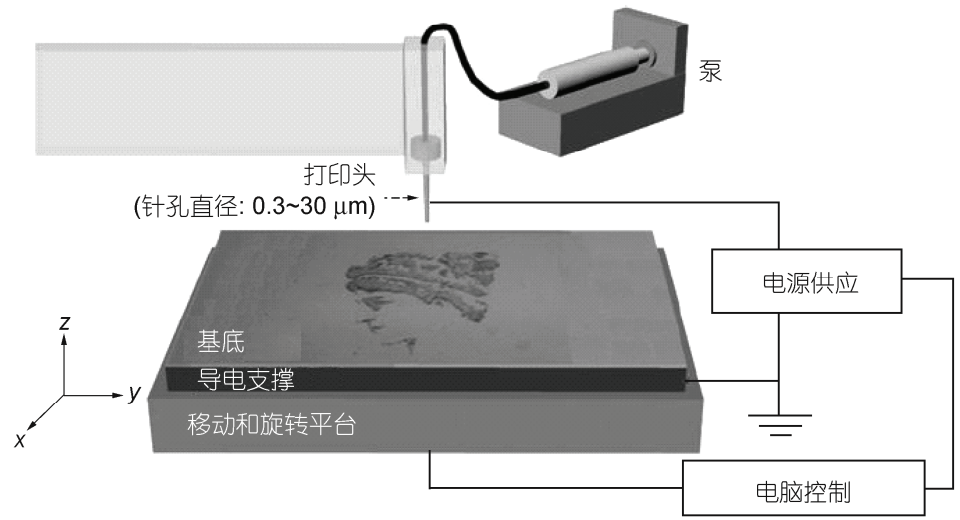

图 24 喷嘴结构和高精度电喷印系统示意图 ${ }^{[85]}$

(a) 金涂层玻璃毛细管喷嘴的 SEM 图片, 内径为 $2 \mu \mathrm{m}$; (b) 喷嘴与基体的喷配置; (c) 喷印平台示意图 
温栅极绝缘层, 以降低晶体管的工作电压. 其墨液由 单分散相、直径 2 3 $\mathrm{nm}$ 的 $\mathrm{Au}$ 纳米颗粒组成, 喷嘴由 内部亲水外部疏水的玻璃毛细管制成(尖端直径小于 $1 \mu \mathrm{m})$. 亚飞升液滴精度打印可明显降低炦烧温度, 避免有机机半导体的损伤. Lee 等人 ${ }^{[56]}$ 采用直径 180 $\mu \mathrm{m}$ 的喷嘴产生微米级射流, 制作出银导线并测试了 其电学性质. Choi 等人 ${ }^{[109]}$ 研究了脉冲射流模式下的 射流直径与喷嘴尺寸、电场之间的关系，发现基频与 电场强度成正比. Choi 等人 ${ }^{[93]}$ 测试了各种偏置电压 和脉冲信号下的射流性能, 以确定最优的射流条件. 为提高雉射流模式的输出和稳定性, Lee 等人 ${ }^{[110]}$ 利用 硅晶片开发了每个喷嘴可独立控制的多喷嘴电流体 动力喷印系统, 以减少电场的干涉和扭曲.

（V）工艺控制。EHD 喷印过程十分复杂，打印 的微结构和形貌受以下因素的影响 ${ }^{[85 ~ 86,92,98,103,105,111]}$ : （1）材料因素: 包括材料结构(分子量、分子链类型、 分子链长度等)、溶液物理性质(黏度、杨氏模量、电 导率和介电常数等)等; (2) 工艺因素: 包括控制参数 (喷嘴与收集电极的距离、电压、喷嘴直径等)、环境 参数(压力、湿度、温度等)等. 为了获得稳定的射流 实现柔性电子器件高精度制造, 必须对以上因素进 行有效控制 ${ }^{[78]}$.

由于电荷间斥力和空气扰动引起的干扰，射流/ 液滴/纤维的定向控制十分困难. 在电喷印和电喷涂 中, 射流或液滴飞抵基板时仍为液态; 电喷涂将液滴 雾化形成薄膜; 电喷印通过控制单一液滴形成特定 图案, 其操控更加复杂. 在电喷涂研究方面, Jaworek 和 $\mathrm{Krupa}^{[96]}$ 利用屏蔽电极获得更加均匀的电场以提 高电喷涂的操控性; Kim 等人 ${ }^{[12]}$ 利用高温玻璃导管 使液滴集中到基板表面, 在管内通人加热的保护气 体获得高度均匀的薄膜. 为避免电喷印射流的不稳 定性, 可减少喷嘴与收集电极间距离和喷嘴直径, 或 设计新的喷嘴结构 ${ }^{[93,110]}$, 如 Sorensen ${ }^{[113]}$ 设计了一个 中心带有尖锐电极、倒置喇叭形状的不锈钢喷嘴. 对 于电纺丝, 射流飞抵基板时为固态, 其控制目标是得 到定向的纳米纤维及其组装体，主要控制手段包括：

(1) 动态机械装置 ${ }^{[22]}$ : 如滚筒、静电板和附加拉伸等;

（2）电场调控 ${ }^{[2]}$ : 使用不同形状和位置的辅助电极;

(3) 近场操控 ${ }^{[95]}$ : 减小电极间的距离和电压.

为实现不同线宽图案的 EHD 喷印, 需要对其断 裂过程及其机理进行研究与控制 ${ }^{[56]}$. 目前已获得的 研究结论包括: (1) 更大分子量的物质溶解导致溶液
黏性会增加, 一定程度的黏性有利于电纺出连续的 纳米纤维, 但不利于电喷涂和电喷印中溶液的断裂; （2）表面张力影响液体的比表面积, 在电喷印和电喷 涂中表面张力增大对小液滴的形成有益, 而在纺丝 中则会形成液珠；(3) 当溶液电导率增大，射流速度 也会增大, 拉伸力增强, 可得到直径更小的液滴和纤 维; (4) 介电常数直接影响表面电荷密度, 对于电纺 丝将增强其“鞭动”行为使得纤维变细和定位更难, 对于电喷印和电喷涂将降低液滴的直径而增加不稳 定性；(5) 喷嘴处溶液的高速流动会增加射流直径, 层的沉积速度与其最终厚度成比例关系 ${ }^{[86]}$; (6) 温度 与压力、黏性相关, 提高温度将增加蒸发的速度并降 低液体的黏度; (7) 直径越小的喷嘴可获得越高的分 辨率; (8) 电极间距直接影响飞行时间和电场强度, 较大间距可生成细小的液滴和纤维, 但增加不稳定 性；（9）控制电场可减小液滴尺寸，但其作用过程十 分复杂 ${ }^{[94]}$, 同时影响液滴和纤维的形貌、分辨率和稳 定性. 此外, 膜的厚度、结晶度、质地和沉积速度都可 通过调节电压、流速、溶液浓度和基板温度进行控制 ${ }^{[86]}$.

\section{4 喷印装置与设备}

应用一般打印设备可以直接打印出不同尺寸的 柔性电子器件, 但直到现在还没有出现商品化的 EHD 喷印设备. 为研制高效高精度喷印设备, 须解决工艺、 材料等参数不同层次优化与控制问题. 首先, 须协调 基板的传输速度、模板数据处理速度、压电驱动器的 触发速度等, 并对柔性基板的畸变进行补偿 ${ }^{[114]}$. 其 次，须优化墨液黏性、表面张力以及喷嘴尺寸 ${ }^{[26,34]}$ 等影响喷射墨滴尺寸的参数. 最后, 须优化喷嘴、墨 液和基板的物理化学性能以确保墨液的打印性(包括 墨液可喷射、喷嘴不堵塞、墨滴直径均匀等 $)^{[78]}$.

\section{1 传统故印设备}

目前, 普通的桌面彩色喷墨打印机已用于打印 聚合物溶液, 如制作了 PZT 柱(IBM Colorjet $)^{[115]}$, PAH/PAA 层(Epson Stylus Color 670) ${ }^{[116]}$, 导电图案 层(Cannon BJC 4550) $)^{[117]}$, OLED (HP 5550) $)^{[118]}$ 等, 所 用打印设备的性能见表 4. 普通喷墨打印机的分辨率 大约 $20 \mu \mathrm{m}$, 不适用于聚合物和纳米粒子的打印. 最 近, 点胶工艺开始应用于柔性电子器件制造, 如包装 中气溶胶沉积等. 表 5 给出了部分聚合物点胶设备型 号及其性能参数, Litrex, Nano-plotter, GeSim 等仪 
表 4 普通桌面喷印设备

\begin{tabular}{ccccc}
\hline 设备 & $\begin{array}{c}\text { 标准工作 } \\
\text { 空间 }\end{array}$ & $\begin{array}{c}\text { 打印速率/ } \\
\mathrm{ppm}\end{array}$ & 工作温度 & $\begin{array}{c}\text { 最高分辨率 } \\
/ \mathrm{dpi}\end{array}$ \\
\hline $\begin{array}{c}\text { Epson Stylus } \\
\text { Color 670 }\end{array}$ & $\mathrm{A} 4$ & 5 & 室温 & $720 \times 1440 \mathrm{dpi}$ \\
$\begin{array}{c}\text { Cannon BJC 4550 } \\
\begin{array}{c}\text { Hewlett Packard } \\
\text { (HP 5550) }\end{array}\end{array}$ & $\mathrm{A} 4$ & 5 & 室温 & $720 \times 360 \mathrm{dpi}$ \\
\hline
\end{tabular}

器公司也可提供类似微滴打印系统. 喷墨点胶设备 设计需考虑的关键因素：(1) 喷嘴设计：为实现 1 皮 升以下墨滴的点胶, 需采用内径小于 $20 \mu \mathrm{m}$ 的喷嘴. (2) $X Y Z$ 运动平台控制: 利用滚珠丝杜和伺服系统进 行控制可实现平台运动定位精度 $\pm 5 \mu \mathrm{m}$, 采用直线电 机可进一步提高定位精度 ${ }^{[119]}$.

\subsection{EHD 喷印实验装置}

（i ）EHD 喷印实验装置. 目前，搭建 EHD 喷印 平台的最大挑战是如何控制墨液流动速率和高压电 场以实现墨滴均匀的雉形喷射. 如图 24 所示, Roger 等 ${ }^{[85}$ 搭建的 EHD 喷印平台包括：精密流量泵(流率 $\leqslant$ $30 \mathrm{pL} \cdot \mathrm{s}^{-1}$ )或气压控制器(压力 $\leqslant 5 \mathrm{psi}$ )、镀金喷嘴、高
压电源，以及固定在 5 轴运动平台上的基板/电极. 通 过上述平台研究了喷嘴内径与墨滴直径、线宽之间的 关系, 实现了典型电路图案的金属互联、电极和探测 点等高分辨率喷印, 制造出特征尺寸为 $1 \mu \mathrm{m}$ 的功能 晶体管. Chen 等人 ${ }^{[120]}$ 建立了用于分析电脉冲喷射行 为的实验验证装置. 为减少电场中的干涉和扭曲, Lee 等人 ${ }^{[110]}$ 开发了可独立控制的多喷嘴装置(图 25), 由 3 个内径为 $360 \mu \mathrm{m}$ 、外径为 $1100 \mu \mathrm{m}$ 、相邻间距 为 $2100 \mu \mathrm{m}$ 的喷嘴组成. 喷嘴内壁涂有防银纳米胶 溶液浸润的聚四氟乙烯, 能形成稳定的对称雉形喷 射，可有效提高多层沉积定位精度.

（ii）电喷涂实验装置. 电喷涂最近被应用于制 作柔性电子器件, 已开发出一些实验装置, 如多喷嘴 电喷涂系统 ${ }^{[121]}$. 在图 26 所示的电喷涂装置中, 玻璃 毛细管的最小直径为 $20 \mu \mathrm{m}$, 与玻璃 ITO 基板的距离 为 $4 \mathrm{~cm}$, 高压电源最大供电 $5 \mathrm{kV}$, 以及丙烯酸腔为 $20 \mathrm{~cm} \times 20 \mathrm{~cm} \times 15 \mathrm{~cm}$. 使用 $2.5 \sim 4 \mathrm{kV}$ 的喷射电压沉 积出厚 $10 \sim 200 \mathrm{~nm}$ 宽 $100 \mu \mathrm{m}$ 薄膜, 成功制作出 OLED 器件 ${ }^{[122]}$.

(iii）电纺丝实验装置. 如图 27 所示, IMET 公司

表 5 聚合物点胶喷印设备

\begin{tabular}{|c|c|c|c|c|c|}
\hline 设备 & 工作空间 $/ \mathrm{mm} \times \mathrm{mm}$ & 精确度 $/ \mu \mathrm{m}$ & 墨滴大小/pL & 喷嘴 & 频率 $/ \mathrm{kHz}$ \\
\hline Microfab Jetlab-II & $\begin{array}{c}150 \times 150,200 \times 200 \\
\text { Option: custom }\end{array}$ & $\begin{array}{c} \pm 15 \text { Option: } \\
\pm 3 \text { with maping }\end{array}$ & $5 \sim 500$ & 4 & 20 \\
\hline Microfab Jetlab-4 & $\begin{array}{c}160 \times 120,210 \times 275 \\
\text { Option: custom }\end{array}$ & $\begin{array}{c} \pm 30, \\
\text { 无方向 }\end{array}$ & $5 \sim 500$ & 4 & 20 \\
\hline FUJIFILM DMP-3000 & $300 \times 300$ & \pm 5 & $1 \sim 10$ & 128 & $10 / 15$ \\
\hline LP50 inkjet printer & A4 & \pm 5 & $12 \sim 25$ & 128 & 25 \\
\hline Microdrop, MD-P-802+AD-K-401 & $200 \times 200$ & \pm 5 & $20 \sim 180$ & 1 & 20 \\
\hline
\end{tabular}

(a)

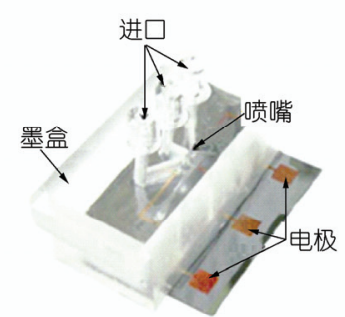

(b)

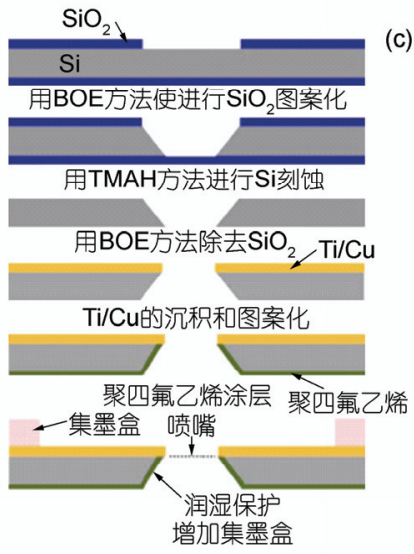

(c)

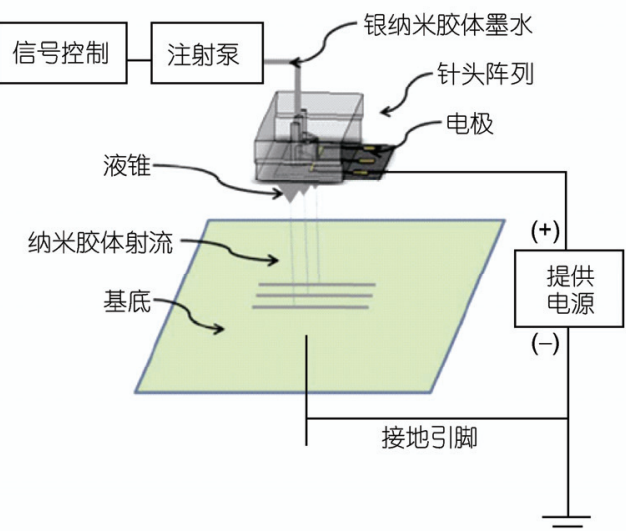

图 25 多喷嘴及 EHD 喷印系统结构图 ${ }^{[110]}$

(a) 多喷嘴陈列; (b) 多喷嘴制备流程图; (c) EHDP 系统结构图 

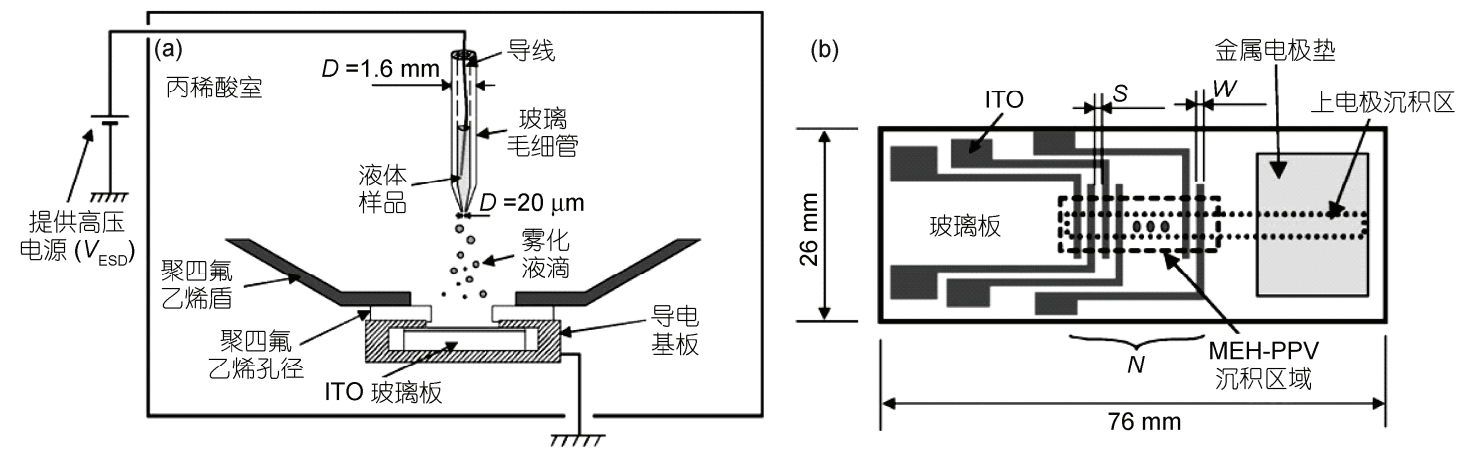

图 26 电喷射沉积法制造 OLED 薄膜的装置

(a) 装置组成; (b) 用于沉积的基板布局

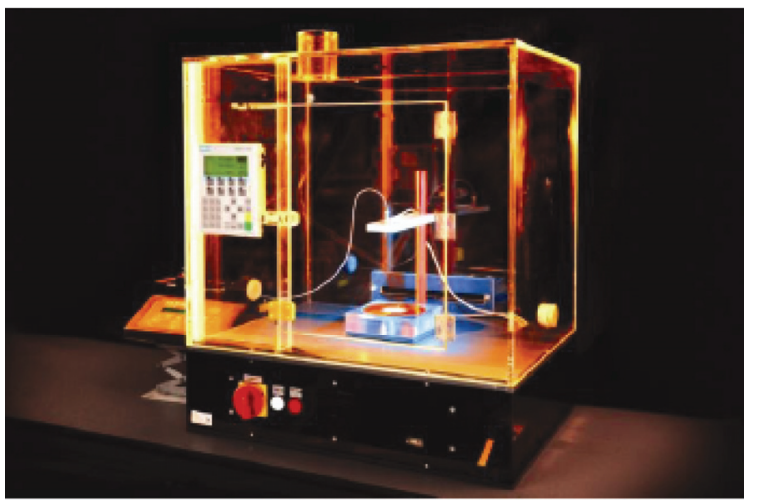

图 27 IMET 电纺丝设备

http://www.imetechnologies.nl/

开发应的电纺丝系统主要包括透明的环境控制室(可 控的温度范围 $15 \sim 50^{\circ} \mathrm{C}$, 湿度范围 $10 \%$ 90\%)、高压 电源及其控制模块、精密流量洜、喷嘴以及收集平台 等. 目前正在研究如何通过预制二维或三维结构的 电极和丝网收集器实现纤维的高精度定向.

如图 28 和表 6 所示, Elmarco 公司已制造出用于 高质量纳米纤维生产的系列化设备: NS Lab(用于新
颖聚合物纺丝工艺研究)、NS Pilot Line(用于新产品 开发试验生产)和 NS line(用于规模化生产), 采用鼓 膜收集器有效解决了纤维无序问题. 此外, 采用多喷 头 ${ }^{[123,124]}$ 可实现纳米纤维高效生产.

\section{3 卷到卷制造工艺}

按照基板进给方式, 柔性电子器件制造工艺可 分为非连续式(片式制造工艺)和卷到卷式. 卷到卷制 造工艺充分利用柔性基板的特性, 通过转轴带动柔 性基板实现柔性电子高效、高可靠的制造，因而更适 合柔性电子大规模生产 ${ }^{[93]}$. 卷到卷制造设备成本与 基板宽度有关, 而与器件面积大小无关 ${ }^{[1]}$, 尤其适合 于大面积柔性电子器件制造.

目前, 已初步开发出集成卷到卷的柔性电子制 造装置. 在图 29 所示的显示器件卷到卷制造 ${ }^{[125]}$ 过程 中, 顶层薄膜采用真空喷射, 底层薄膜先后经历苯胺 印制、烘干、真空喷射、清除、凹版印刷等, 然后通 过碾压实现不同薄膜层复合, 输送的薄膜厚度为 500 $\mathrm{nm} 2 \mu \mathrm{m}$. 图 30 所示的卷到卷纳米压印系统 ${ }^{[126]}$ 包含
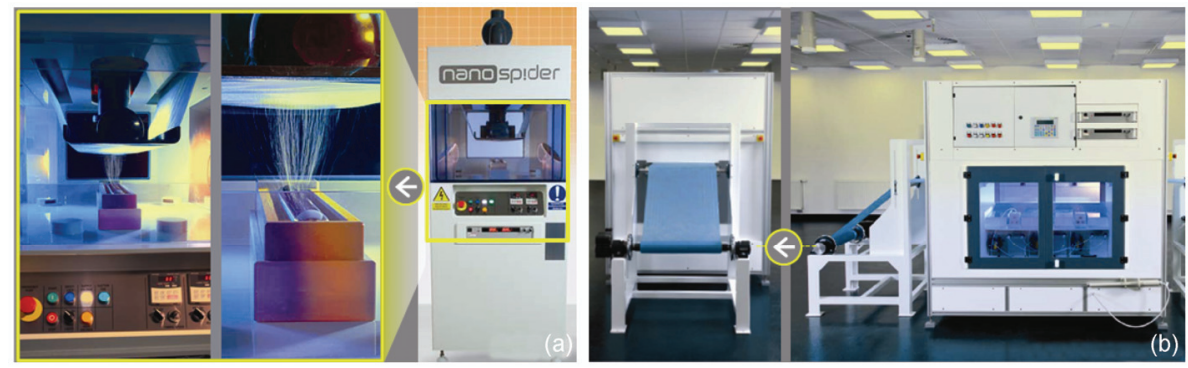

图 28 NS Lab(a)和 NS Pilot Line 系统(b)

http://www.elmarco.com/ 
表 6 NS Lab 和 NS Pilot Line 系统

\begin{tabular}{cccccccc}
\hline 型号 & 电压 & 收集器高度 & $\begin{array}{c}\text { 基座速度 } \\
/ \mathrm{mm}\end{array}$ & $\begin{array}{c}\text { 电极旋转速度 } \\
/ \mathrm{m} \cdot \mathrm{min}^{-1}\end{array}$ & $\begin{array}{c}\text { 产能 } \\
/ \mathrm{g} \cdot \mathrm{min}^{-1}\end{array}$ & $\begin{array}{c}\text { 工作环境 } \\
/{ }^{\circ} \mathrm{C}\end{array}$ & $\begin{array}{c}\text { 最小纤维直径 } \\
/ \mathrm{nm}\end{array}$ \\
\hline NS Lab & $0 \sim 80$ & $60 \sim 210$ & $0.10 \sim 1.56$ & $1 \sim 16$ & 1 & $18 \sim 30$ & $80 \times(1 \pm 30 \%)$ \\
NS Pilot & $0 \sim 80^{\mathrm{a})}, 0 \sim 60^{\mathrm{a})}$ & $100 \sim 250$ & $0.20 \sim 5$ & $1 \sim 16$ & 3 & $18 \sim 40$ & $80 \times(1 \pm 30 \%)$ \\
\hline
\end{tabular}

a) 纺纱头阳极 $(0 \sim 80 \mathrm{kV})$, 采集阴电极 $(0 \sim 60 \mathrm{kV})$

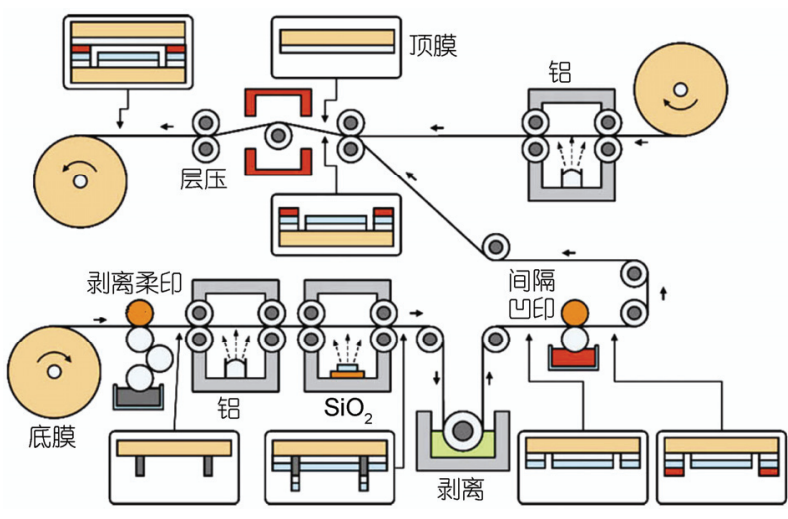

图 29 发展中的卷到卷制造系统 ${ }^{[125]}$

涂层、压印和金属沉积工艺单元，可实现特征尺寸小 于 $70 \mathrm{~nm}$ 的聚合物图案化. 图 31 所示的卷到卷刻蚀 系统 ${ }^{[127]}$ 采用了集成光学显微镜和激光干涉仪的精 密光学测量系统, 在柔性基板上实现连续、高分辨率 图案化, 其中分辨率达 $10 \mu \mathrm{m}$ (最高达 $1 \mu \mathrm{m}$ )、层间套 准精度 $\pm 2.5 \mu \mathrm{m}$ (最高达 $\pm 0.3 \mu \mathrm{m}$ ). 其他传统的平板 印刷、苯胺印刷、真空涂层和纳米压印等图案化工艺
也实现与卷到卷技术集成 ${ }^{[4,93]}$.

为实现高分辨、高效、高可靠地卷到卷喷印制造, 须对薄膜基板的变形进行有效控制, 需研究张力控 制、浮动辊补偿、层与层套准、机械系统设计等关键 技术. 在 EHD 喷印要求的超薄柔性基板(小于 $1 \mu \mathrm{m}$ 薄膜)卷到卷间歇式高速进给过程中, 受输送系统料 辊几何参数 (如料辊数目、布局、半径、包络角等) 以 及物理特性(表面摩擦力、静电力等)、基板材质不均 引起的弹性模量随机变化、间歇式快速进给运动冲 击、纠偏控制器扰动等多种复杂因素的影响, 存在进 给运动与张力的耦合, 对基板传输和定位精度提出 了挑战.

\section{5 挑战与展望}

柔性电子技术发展不会取代传统的微电子技术, 而将开辟出许多新的电子产品应用领域 ${ }^{[32]}$. 喷印技 术作为一类适合不同材料、不同尺度制造的柔性制造 工艺, 应用多种机理和能量模式实现纳米到毫米范 围的图案化. 为实现柔性电子器件高效高精制造, 喷

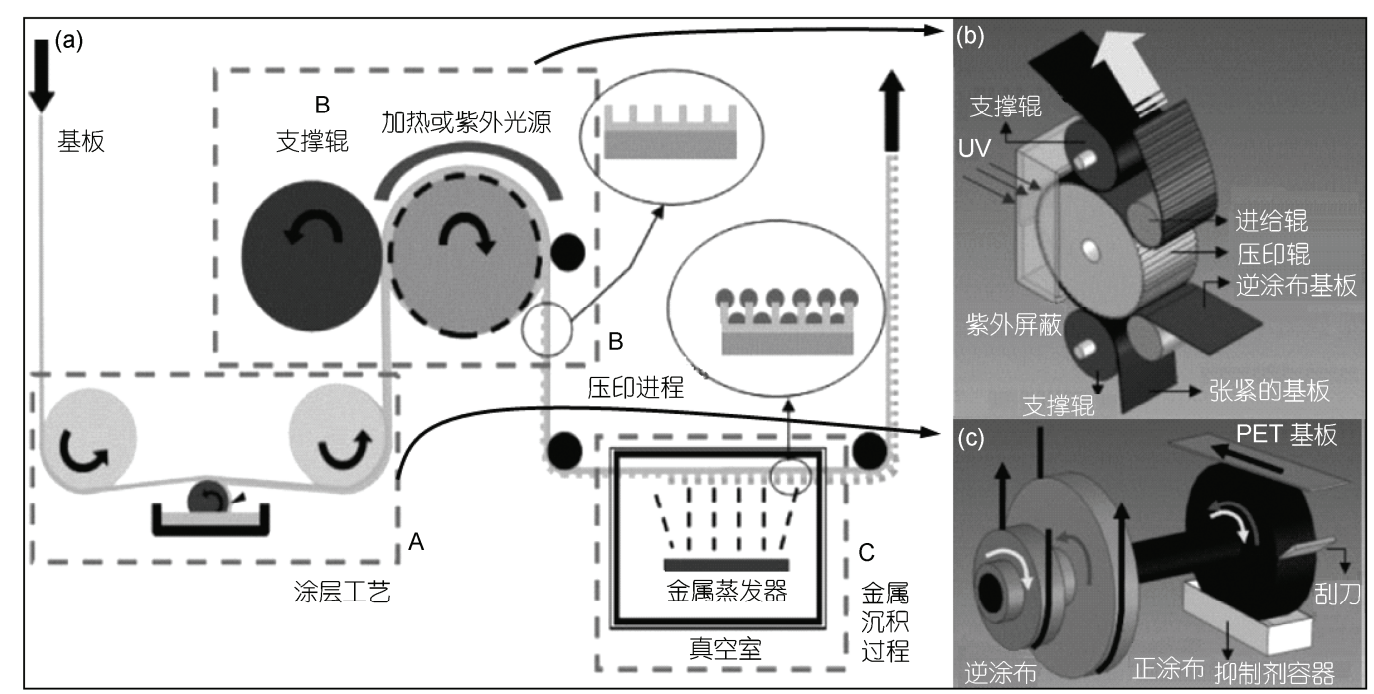

图 30 工业应用前景和挑战

(a) R2RNIL 示意; (b) 印制单元; (c) 涂层单元 ${ }^{[126]}$ 


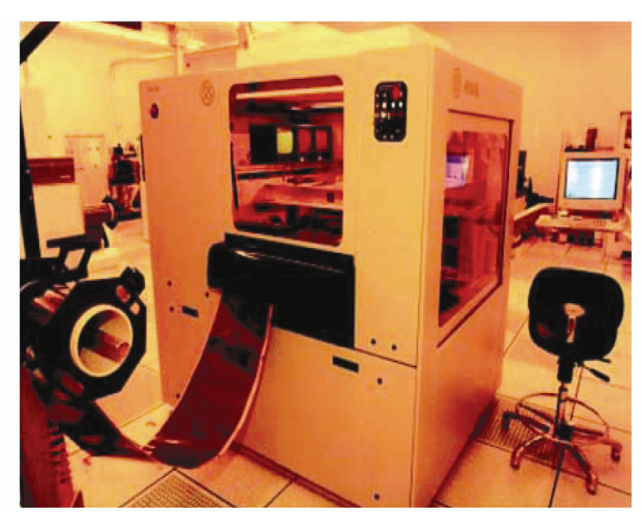

图 31 HexScanTM $3100 \mathrm{SRE}^{[127]}$

印技术还需解决一系列科学技术挑战:

(i ) 液滴操控机理. 在纳米尺度下, 液滴表面/ 界面效应对泰勒雉形成、薄膜沉积、层间界面强度, 甚至对 OTFT 的性能都至关重要; 喷射过程中高压电 场外场作用影响喷印的动态行为 (如固化、形变、弹 道、均匀性等); 使用微粒或颜料墨液易形成类似咖
啡环污渍等非均匀结构. 揭示上述过程的物理化学 作用机理对实现液滴可控、高精喷射十分重要.

(ii) 喷墨打印装置设计. 喷嘴设计和制造是开 发喷印装置的瓶颈之一, 须优化喷嘴的物理化学性 能, 实现对称稳定的圆雉形喷射, 并确保聚合物的可 打印性(包括毛细管内/外部的润湿性、墨液喷射性等)

（iii）喷印功能材料制备. 分子/纳米尺度结构 直接影响墨液以及器件性能, 如形貌、黏附性、机械 集成度、可溶性、化学和环境稳定性等. 需解决的关 键问题包括如何调控墨液的黏度、表面张力、对基 底的附着力等物理和化学属性, 保证器件良好的电 子迁移率、开关电压、阈值电压稳定性和关闭电流 等特性.

(iv) 与卷到卷技术集成. 为实现不同材料不同 结构的高效高精制造, 需将压印、真空沉积、层压等 工艺与卷到卷技术集成, 须研究工艺过程优化、超薄 柔性基板变形与张力控制、精确套准与定位等关键 技术.

\section{参考文献}

1 Wong W S, Salleo A. Flexible Electronics: Materials and Applications. New York: Springer, 2009

2 Reuss R H, Chalamala B R, Moussessian A, et al. Macroelectronics: Perspectives on technology and applications. P IEEE, 2005, 93: 12391256

3 Kim D H, Ahn J H, Choi W M, et al. Stretchable and foldable silicon integrated circuits. Science, 2008, 320: 507-511

4 Crawford G P. Flexible Flat Panel Displays. Chichester: John Wiley \& Sons, Ltd, 2005

5 Jang J. Displays develop a new flexibility. Mater Today, 2006, 9: 46-52

6 Krebs F C. Fabrication and processing of polymer solar cells: A review of printing and coating techniques. Sol Energy Mater Sol Cells, 2009, 93: 394-412

7 Mayer A C, Scully S R, Hardin B E, et al. Polymer-based solar cells. Mater Today, 2007, 10: 28-33

8 Someya T, Kato Y, Sekitani T, et al. Conformable, flexible, large-area networks of pressure and thermal sensors with organic transistor active matrixes. Proc Natl Acad Sci USA, 2005, 102: 12321-12325

9 Madden P G A. Development and modeling of conducting polymer actuators and the fabrication of a conducting polymer based feedback loop. Cambridge: Massachusetts Institute of Technology, 2003

10 Kim D H, Rogers J A. Stretchable electronics: Materials strategies and devices. Adv Mater, 2008, 20: 4887—4892

11 Park S I, Ahn J H, Feng X, et al. Theoretical and experimental studies of bending of inorganic electronic materials on plastic substrates. Adv Funct Mater, 2008, 18: 2673-2684

12 Khang D Y, Jiang H, Huang Y, et al. A stretchable form of single-crystal silicon for high-performance electronics on rubber substrates. Science, 2006, 311: 208-212

13 Jiang H, Khang D Y, Song J, et al. Finite deformation mechanics in buckled thin films on compliant supports. Proc Natl Acad Sci USA, 2007, 104: 15607-15612

14 Lacour S P, Chan D, Wagner S, et al. Mechanisms of reversible stretchability of thin metal films on elastomeric substrates. Appl Phys Lett, 2006, 88: 204103

15 Sun Y, Choi W M, Jiang H, et al. Controlled buckling of semiconductor nanoribbons for stretchable electronics. Nat Nanotech, 2006, 1 : $201-206$

16 Huang Z Y, Hong W, Suo Z. Nonlinear analyses of wrinkles in a film bonded to a compliant substrate. J Mech Phys Solids, 2005, 53: 21012118 
17 Jiang H, Sun Y, Rogersc J A, et al. Post-buckling analysis for the precisely controlled buckling of thin film encapsulated by elastomeric substrates. Int J Solids Struct, 2008, 45: 2014-2023

18 Wagner S, Lacour S P, Jones J, et al. Electronic skin: Architecture and components. Physica E, 2004, 25: 326-334

19 Gleskova H, Cheng I C, Wagner S, et al. Mechanics of thin-film transistors and solar cells on flexible substrates. Sol Energy, 2006, 80: $687-693$

20 Lacour S P, Wagner S, Huang Z, et al. Stretchable gold conductors on elastomeric substrates. Appl Phys Lett, 2003, 82: 2404-2406

21 许巍, 卢天健. 柔性电子系统及其力学性能. 力学进展, 2008, 38: 137-150

22 Huang Y, Yin Z, Xiong Y. Thermomechanical analysis of stiff films on compliant substrates with temperature-depended material properties. J Appl Mech-T ASME, 2010, 77: 1-9

23 Huang Y, Yin Z, Xiong Y. Thermomechanical analysis of thin films on temperature-dependent elastomeric substrates in flexible heterogeneous electronics. Thin Solid Films, 2010, 518: 1698-1702

24 Logothetidis S. Flexible organic electronic devices: Materials, process and applications. Mater Sci Eng B, 2008, 152: 96-104

25 de Gans B J, Duineveld P C, Schubert U S. Inkjet printing of polymers: State of the art and future developments. Adv Mater, 2004, 16: $203-213$

26 Menard E, Meitl M A, Sun Y G, et al. Micro- and nanopatterning techniques for organic electronic and optoelectronic systems. Chem Rev, 2007, 107: 1117-1160

27 Calvert P. Inkjet printing for materials and devices. Chem Mater, 2001, 13: 3299-3305

28 兰红波, 丁玉成, 刘红忠, 等. 纳米压印光刻模具制作技术研究进展及其发展趋势. 机械工程学报, 2008, 45: 1-13

29 Sirringhaus H, Kawase T, Friend R H, et al. High-resolution inkjet printing of all-polymer transistor circuits. Science, 2000, 290: 21232126

30 Wang Y, Bokor J, Lee A. Maskless lithography using drop-on-demand inkjet printing method. P Soc Photo-Opt Ins, 2004, 5374: 628636,1110

31 Singh T B, Sariciftci N S. Progress in plastic electronics devices. Annu Rev Mater Res, 2006, 36: 199-230

32 Kelley T W, Baude P F, Gerlach C, et al. Recent progress in organic electronics: materials, devices, and processes. Chem Mater, 2004, 16: $4413-4422$

33 Reichmanis E, Katz H, Kloc C, et al. Plastic electronic devices: From materials design to device applications. Bell Labs Tech J, 2005, 10: 87-105

34 Jang D, Kim D, Moon J. Influence of fluid physical properties on ink-jet printability. Langmuir, 2009, 25: 2629-2635

35 Bergeron V, Bonn D, Martin J Y, et al. Controlling droplet deposition with polymer additives. Nature, 2000, 405: 772-775

36 Martin G D, Hoath S D, Hutchings I M. Inkjet printing-the physics of manipulating liquid jets and drops. J Phys: Conf Ser, 2008, 105: $012001-012014$

37 de Gans B J, Schubert U S. Inkjet printing of polymer micro-arrays and libraries: Instrumentation, requirements, and perspectives. Macromol Rapid Comm, 2003, 24: 659-666

38 Mabrook M F, Pearson C, Jombert A S, et al. The morphology, electrical conductivity and vapour sensing ability of inkjet-printed thin films of single-wall carbon nanotubes. Carbon, 2009, 47: 752-757

39 Fan Z J, Wei T, Luo G H, et al. Fabrication and characterization of multi-walled carbon nanotubes-based ink. J Mater Sci, 2005, 40: 50755077

40 Wei T, Ruan J, Fan Z J, et al. Preparation of a carbon nanotube film by ink-jet printing. Carbon, 2007, 45: 2712-2716

41 Song J W, Kim Y S, Yoon Y H, et al. The production of transparent carbon nanotube field emitters using inkjet printing. Phys E, 2009, 41: $1513-1516$

42 Dror Y, Salalha W, Khalfin R L, et al. Carbon nanotubes embedded in oriented polymer nanofibers by electrospinning. Langmuir, 2003 , 19: $7012-7020$

43 Zhang Q H, Chang Z J, Zhu M F, et al. Electrospun carbon nanotube composite nanofibres with uniaxially aligned arrays. Nanotechnology, 2007, 18: 115611-115616

44 Magdassi S, Bassa A, Vinetsky Y, et al. Silver nanoparticles as pigments for water-based ink-jet inks. Chem Mater, 2003, 15: 2208-2217

45 Lee H H, Chou K S, Huang K C. Inkjet printing of nanosized silver colloids. Nanotechnology, 2005, 16: 2436-2441

46 Perelaer J, de Gans B J, Schubert U S. Ink-jet printing and microwave sintering of conductive silver tracks. Adv Mater, 2006, 18: 21012104

47 Dearden A L, Smith P J, Shin D Y, et al. A low curing temperature silver ink for use in ink-jet printing and subsequent production of conductive tracks. Macromol Rapid Comm, 2005, 26: 315-318

48 Liu Z, Wen F S, Li W L. Synthesis and electroluminescence properties of europium(III) complexes with new second ligands. Thin Solid 
Films, 2005, 478: 265-270

49 Park B K, Kim D, Jeong S, et al. Direct writing of copper conductive patterns by ink-jet printing. Thin Solid Films, 2007, 515: 77067711

50 Woo K, Kim D, Kim J S, et al. Ink-jet printing of Cu-Ag-based highly conductive tracks on a transparent substrate. Langmuir, 2009, 25: $429-433$

51 Kamyshny A, Ben-Moshe M, Aviezer S, et al. Ink-jet printing of metallic nanoparticles and microemulsions. Macromol Rapid Comm, 2005, 26: 281-288

52 Kim D, Moon J. Highly conductive ink jet printed films of nanosilver particles for printable electronics. Electrochem Solid ST, 2005, 8: $\mathrm{J} 30-\mathrm{J} 33$

53 Hsu S L C, Wu R T. Synthesis of contamination-free silver nanoparticle suspensions for micro-interconnects. Mater Lett, 2007, 61: 37193722

54 Wu R T, Hsu S L C. Preparation of highly concentrated and stable suspensions of silver nanoparticles by an organic base catalyzed reduction reaction. Mater Res Bull, 2008, 43: 1276-1281

55 Wu J T, Hsu S L C, Tsai M H, et al. Conductive silver patterns via ethylene glycol vapor reduction of ink-jet printed silver nitrate tracks on a polyimide substrate. Thin Solid Films, 2009, 517: 5913-5917

56 Lee D Y, Shin Y S, Park S E, et al. Electrohydrodynamic printing of silver nanoparticles by using a focused nanocolloid jet. Appl Phys Lett, 2007, 90: 0819051-0819053

57 Lee D Y, Lee J C, Shin Y S, et al. Structuring of conductive silver line by electrohydrodynamic jet printing and its electrical characterization. J Phys: Conf Ser, 2007, 142: 012039

58 Chronakis I S, Grapenson S, Jakob A. Conductive polypyrrole nanofibers via electrospinning: Electrical and morphological properties. Polymer, 2006, 47: 1597-1603

59 Shen W F, Zhao Y, Zhang C B. The preparation of ZnO based gas-sensing thin films by ink-jet printing method. Thin Solid Films, 2005, 483: $382-387$

60 Choi J H, Khang D Y, Myoung J M. Fabrication and characterization of ZnO nanowire transistors with organic polymer as a dielectric layer. Solid State Commun, 2008, 148: 126-130

61 Yang Y J, Jiang Y D, Xu J H, et al. Conducting PEDOT-PSS composite films assembled by LB technique. Colloid Surface A, 2007, 302: $157-161$

62 Jang J, Chang M, Yoon H. Chemical sensors based on highly conductive poly(3,4-ethylenedioxythiophene) nanorods. Adv Mater, 2005, 17: $1616-1620$

63 Ballarin B, Fraleoni-Morgera A, Frascaro D, et al. Thermal inkjet microdeposition of PEDOT: PSS on ITO-coated glass and characterization of the obtained film. Synth Met, 2004, 146: 201-205

64 Eom S H, Senthilarasu S, Uthirakumar P, et al. Polymer solar cells based on inkjet-printed PEDOT:PSS layer. Org Electron, 2009, 10: $536-542$

65 Hohnholz D, Okuzaki H, MacDiarmid A G. Plastic electronic devices through line patterning of conducting polymers. Adv Funct Mater, 2005, 15: 51-56

66 Kwon I W, Son H J, Kim W Y, et al. Thermistor behavior of PEDOT: PSS thin film. Synth Met, 2009, 159: 1174-1177

67 Jeong S, Kim D, Moon J. Ink-jet-printed organic-inorganic hybrid dielectrics for organic thin-film transistors. J Phys Chem C, 2008, 112: $5245-5249$

68 Xie X L, Mai Y W, Zhou X P. Dispersion and alignment of carbon nanotubes in polymer matrix: A review. Mat Sci Eng R, 2005, 49: 89112

69 Lin H W, Hwu W H, Ger M D. The dispersion of silver nanoparticles with physical dispersal procedures. J Mater Process Tech, 2008, 206: $56-61$

70 Hon K K B, Li L, Hutchings I M. Direct writing technology-Advances and developments. Cirp Ann-Manuf Techn, 2008, 57: 601-620

71 Le H P. Progress and trends in ink-jet printing technology. J Imag Sci Tech, 1998, 42: 49-62

72 Paul K E, Wong W S, Ready S E, et al. Additive jet printing of polymer thin-film transistors. Appl Phys Lett, 2003, 83: 2070-2072

73 Wang Y, Bokor J. Ultra-high-resolution monolithic thermal bubble inkjet print head. J Micro-Nanolith Mem, 2007, 6: 043009

74 Dong H M, Carr W W, Morris J F. An experimental study of drop-on-demand drop formation. Phys Fluids, 2006, 18: 0721021—0721016

75 Noguchi Y, Sekitani T, Yokota T, et al. Direct inkjet printing of silver electrodes on organic semiconductors for thin-film transistors with top contact geometry. Appl Phys Lett, 2008, 93: 0433031—0433033

76 Duineveld P C. The stability of ink-jet printed lines of liquid with zero receding contact angle on a homogeneous substrate. J Fluid Mech, 2003, 477: 175-200 
77 Lim J A, Lee H S, Lee W H, et al. Control of the morphology and structural development of solution-processed functionalized acenes for high-performance organic transistors. Adv Funct Mater, 2009, 19: 1515-1525

78 Roy S. Fabrication of micro- and nano-structured materials using mask-less processes. J Phys D Appl Phys, 2007, 40: R413-R426

79 Sirringhaus H, Kawase T, Friend R H. High-resolution ink-jet printing of all-polymer transistor circuits. MRS Bull, 2001, 26: 539-543

80 Li S P, Newsome C J, Kugler T, et al. Polymer thin film transistors with self-aligned gates fabricated using ink-jet printing. Appl Phys Lett, 2007, 90: 1721031-1721033

81 Noh Y Y, Zhao N, Caironi M, et al. Downscaling of self-aligned, all-printed polymer thin-film transistors. Nat Nanotechnol, 2007, 2: $784-789$

82 Bao Z N. Fine printing. Nat Mater, 2004, 3: 137-138

83 Sele C W, von Werne T, Friend R H, et al. Lithography-free, self-aligned inkjet printing with sub-hundred-nanometer resolution. Adv Mater, 2005, 17: 997-1001

84 Ko S H, Pan H, Grigoropoulos C P, et al. All-inkjet-printed flexible electronics fabrication on a polymer substrate by low-temperature high-resolution selective laser sintering of metal nanoparticles. Nanotechnology, 2007, 18: 3452021-3452028

85 Park J U, Hardy M, Kang S J, et al. High-resolution electrohydrodynamic jet printing. Nat Mater, 2007, 6: 782-789

86 Jaworek A, Sobczyk A T. Electrospraying route to nanotechnology: An overview. J Electrostat, 2008, 66: 197-219

87 Kim J, Oh H, Kim S S. Electrohydrodynamic drop-on-demand patterning in pulsed cone-jet mode at various frequencies. J Aerosol Sci, 2008, 39: $819-825$

88 Basaran O A. Small-scale free surface flows with breakup: Drop formation and emerging applications. Aiche J, 2002, 48: 1842-1848

89 Jaworek A, Krupa A. Classification of the modes of EHD spraying. J Aerosol Sci, 1999, 30: 873-893

90 Paine M D, Alexander M S, Smith K L, et al. Controlled electrospray pulsation for deposition of femtoliter fluid droplets onto surfaces. J Aerosol Sci, 2007, 38: 315-324

91 Li J L. On the meniscus deformation when the pulsed voltage is applied. J Electrostat, 2006, 64: 44-52

92 Teo W E, Ramakrishna S. A review on electrospinning design and nanofibre assemblies. Nanotechnology, 2006, 17: R89-R106

93 Choi J, Kim Y J, Lee S, et al. Drop-on-demand printing of conductive ink by electrostatic field induced inkjet head. Appl Phys Lett, 2008, 93: $1935081-1935083$

94 Reneker D H, Yarin A L. Electrospinning jets and polymer nanofibers. Polymer, 2008, 49: 2387-2425

95 Jayasinghe S N, Dorey R A, Edirisinghe M J, et al. Preparation of lead zirconate titanate nano-powder by electrohydrodynamic atomization. Appl Phys A Mater, 2005, 80: 723-725

96 Jaworek A. Electrospray droplet sources for thin film deposition. J Mater Sci, 2007, 42: 266-297

97 Saf R, Goriup M, Steindl T, et al. Thin organic films by atmospheric-pressure ion deposition. Nat Mater, 2004, 3: 323-329

98 Salata O V. Tools of nanotechnology: Electrospray. Curr Nanosci, 2005, 1: 25-33

99 Yu F X, Cui J Z, Ranganathan S, et al. Fundamental differences between spray forming and other semisolid processes. Mat Sci Eng A-Struct, 2001, 304: 621-626

100 陈效鹏, 程久生, 尹协振. 电流体动力学研究进展及其应用. 科学通报, 2003, 48: 637-646

101 Fujihara K, Kumar A, Jose R, et al. Spray deposition of electrospun $\mathrm{TiO}_{2}$ nanorods for dye-sensitized solar cell. Nanotechnology, 2007, 18: $3657091-3657095$

102 Cich M, Kim K, Choi H, et al. Deposition of $(\mathrm{Zn}, \mathrm{Mn})(2) \mathrm{SiO}_{4}$ for plasma display panels using charged liquid cluster beam. Appl Phys Lett, 1998, 73: $2116-2118$

103 Huang Z M, Zhang Y Z, Kotaki M, et al. A review on polymer nanofibers by electrospinning and their applications in nanocomposites. Comp Sci Tech, 2003, 63: 2223-2253

104 Yarin A L, Zussman E. Upward needleless electrospinning of multiple nanofibers. Polymer, 2004, 45: 2977-2980

105 Sun D, Chang C, Li S, et al. Near-Field Electrospinning. Nano Lett, 2006, 6: 839-842

106 MacDiarmid A G. "Synthetic metals": A novel role for organic polymers (Nobel lecture). Angew Chem Int Edit, 2001, 40: 2581-2590

107 Jeong J S, Jeon S Y, Lee T Y, et al. Fabrication of MWNTs/nylon conductive composite nanofibers by electrospinning. Diam Relat Mater, 2006, 15: 1839-1843

108 Sekitani T, Noguchi Y, Zschieschang U, et al. Organic transistors manufactured using inkjet technology with subfemtoliter accuracy. Proc Natl Acad Sci USA, 2008, 105: 4976-4980

109 Choi H K, Park J U, Park O O, et al. Scaling laws for jet pulsations associated with high-resolution electrohydrodynamic printing. Appl Phys Lett, 2008, 92: 1231091-1231093

110 Lee J S, Kim S Y, Kim Y J, et al. Design and evaluation of a silicon based multi-nozzle for addressable jetting using a controlled flow rate in electrohydrodynamic jet printing. Appl Phys Lett, 2008, 93: 2431141-2431143 
111 仰大勇, 王洋, 张东舟, 等. 聚碳酸酯静电纺丝微纳结构的形貌控制. 科学通报, 2009, 54: 1161-1166

112 Kim S G, Choi K H, Eun J H, et al. Effects of additives on properties of MgO thin films by electrostatic spray deposition. Thin Solid Films, 2000, 377: 694-698

113 Sorensen G. Ion bombardment of electrosprayed coatings: An alternative to reactive sputtering. Surf Coat Tech, 1999, 112: 221-225

114 Dong H M, Carr W W, Morris J F. Visualization of drop-on-demand inkjet: Drop formation and deposition. Revi Sci Instrum, 2006, 77: 0851011-0851018

115 Bhatti A R, Mott M, Evans J R G, et al. PZT pillars for 1-3 composites prepared by ink-jet printing. J Mater Sci Lett, 2001, 20: 12451248

116 Guo T F, Chang S C, Pyo S, et al. Vertically integrated electronic circuits via a combination of self-assembled polyelectrolytes, ink-jet printing, and electroless metal plating processes. Langmuir, 2002, 18: 8142-8147

117 Kordas K, Mustonen T, Toth G, et al. Inkjet printing of electrically conductive patterns of carbon nanotubes. Small, 2006, 2: 1021-1025

118 Yoshioka Y, Jabbour G E. Desktop inkjet printer as a tool to print conducting polymers. Synth Met, 2006, 156: 779-783

119 Ding H, Xiong Z. Motion stages for electronic packaging design and control. IEEE Robot Autom Mag, 2006, 13: 51-61

120 Chen C H, Saville D A, Aksay I A. Scaling laws for pulsed electrohydrodynamic drop formation. Appl Phys Lett, 2006, 89: 12410311241033

121 Jaworek A, Balachandran W, Lackowski M, et al. Multi-nozzle electrospray system for gas cleaning processes. J Electrostat, 2006, 64: $194-202$

122 Ju J, Yamagata Y, Higuchi T. Thin-film fabrication method for organic light-emitting diodes using electrospray deposition. Adv Mater, 2009, 21: 1-5

123 Darty M A. Methods and apparatus for electrohydrodynamic ejection. US Patent, US 6312110 B1, 2001

124 Lee W S, Jo S M, Go S G, et al. Apparatus of polymer web by electrospinning process. United States Patent, 2003

125 Lo C Y, Hiitola-Keinanen J, Huttunen O H, et al. Novel roll-to-roll lift-off patterned active-matrix display on flexible polymer substrate. Microelectron Eng, 2009, 86: 979-983

126 Ahn S H, Guo L J. High-speed roll-to-roll nanoimprint lithography on flexible plastic substrates. Adv Mater, 2008, 20: 2044—2049

127 Jain K, Klosner M, Zemel M, et al. Flexible electronics and displays: High-resolution, roll-to-roll, projection lithography and photoablation processing technologies for high-throughput production. P IEEE, 2005, 93: 1500-1510 